\title{
An exact solution of linearized flow of an emitting, absorbing and scattering grey gas
}

\author{
By PING CHENG† \\ NASA-Ames Research Center, Moffett Field, California
}

AND A. LEONARD

Stanford University, Stanford, California

(Received 7 October 1969 and in revised form 24 August 1970)

The governing equations for the problem of linearized flow through a normal shock wave in an emitting, absorbing, and scattering grey gas are reduced to two linear coupled integro-differential equations. By separation of variables, these equations are further reduced to an integral equation similar to that which arises in neutron-transport theory. It is shown that this integral equation admits both regular (associated with discrete eigenfunctions) and singular (associated with continuum eigenfunctions) solutions to form a complete set. The exact closed-form solution is obtained by superposition of these eigenfunctions. If the gas downstream of a strong shock is absorption-emission dominated, the discrete mode of the solution disappears downstream. The effects of isotropic scattering are discussed. Quantitative comparison between the numerical results based on the exact solution and on the differential approximation are presented.

\section{Introduction}

The present work is motivated by the desire to obtain an exact solution in the linearized analysis of a problem in radiative gasdynamics, taking into account the effect of scattering. The purpose of this paper is therefore threefold: first, to investigate the effect of scattering in radiative gasdynamies; second, to assess the accuracy of the differential approximation when scattering is taken into consideration; and, third, to provide some exact numerical results as a testing case for solutions based on other approximate techniques in radiative gasdynamics.

The physical problem considered in this paper is that of a plane, stationary shock wave in an inviscid radiating grey gas. The mathematical difficulties of the problem are associated with the fact that governing equations are nonlinear and of integro-differential form (see, for example, Vincenti \& Kruger 1965). The problem has been studied by Clarke (1962), Heaslet \& Baldwin (1963), and Mitchner \& Vinokur (1963), among others. On the basis of the exponential

$\dagger$ Present address: Department of Mechanical Engineering, University of Hawaii, Honolulu, Hawaii 96822. 
approximation (or its equivalent, the differential approximation), these investigators were able to obtain a set of equations of purely differential form from which results can be obtained by numerical integration. In order to assess the accuracy of the exponential approximation, Pearson (1964) has performed numerical integration of the exact non-linear integro-differential equations for this problem and concluded that the exponential approximation is accurate to $1 \%$ in velocity. Later, Vincenti \& Kruger (1965) considered the same problem for the special case when the radiative heat flux is small compared to the convective flux as characterized by large Boltzmann number $(B o)$. Since the effect of the radiation is small for this special case, the governing equations can be linearized with respect to the non-radiating situation. Analytical solutions within the framework of linearized theory and the differential approximation were obtained and expressions for disturbed quantities expanded to the order of 1/Bowere given. All of these investigations have neglected the effect of scattering.

In problems such as the rocket exhaust plume which contains a large amount of particles, the effect of scattering is important (Rochelle 1967, Carlson 1966, Fontenot 1965). In this paper, we shall obtain an exact analytical solution to the same problem considered by Vincenti \& Kruger (1965), taking into consideration the effect of isotropic scattering. Although Vincenti \& Kruger give their solution in the differential approximation to order $1 / B o$, consistent with the linearization, we choose to solve the exact linearized integro-differential equations without further expansion in powers of $1 / B o$ for the purpose of comparing the results numerically with the differential approximation under the same conditions. It will be shown that the governing equations can be combined to yield two coupled linear integro-differential equations. By separation of variables similar to that of the previous paper by the same authors (Cheng \& Leonard 1970), these integro-differential equations are reduced to an integral equation similar to that which arises in neutron-transport theory (Case \& Zweifel 1967). The solution is then obtained in a manner similar to that used in the two-half-space problem considered by Mendelson \& Summerfield (1964).

A closed-form solution is obtained for the disturbed quantities in the flow and radiative fields. Explicit analytic expressions are obtained for the disturbed quantities immediately upstream and downstream from the shock. Quantitative comparisons of the numerical results of the exact solution and the solution based on the differential approximation are made. The effect of isotropic scattering is discussed.

\section{Governing equations and boundary conditions}

Consider a steady normal shock wave located at $x=0$. If the subscript $\infty, j$ denotes conditions at infinity, with $j=1,2$ representing the conditions in front of $(x<0)$ and behind the shock $(x>0)$, respectively, the linearized equations of state, continuity, and momentum for an inviscid planar radiative flow can be combined to give

$$
\frac{d T^{\prime}}{d x}=\frac{u_{\infty, j}}{R}\left[\frac{1}{\gamma M_{\infty, j}^{2}}-1\right] \frac{d u^{\prime}}{d x} \quad(j=1,2)
$$


whereas the equations of energy and state can be combined to yield

$$
\frac{u_{\infty, j} \rho_{\infty, j} \gamma R}{\gamma-1} \frac{d T^{\prime}}{d x}+\rho_{\infty, j} u_{\infty, j}^{2} \frac{d u^{\prime}}{d x}+\frac{d q^{\prime}}{d x}=0 \quad(j=1,2),
$$

where $T^{\prime}, u^{\prime}$, and $q^{\prime}$ are, respectively, the disturbed temperature, velocity, and radiative heat flux; $M_{\infty}, \rho_{\infty}$, and $u_{\infty}$ are the Mach number, density, and velocity at infinity; $\gamma$ and $R$ are the specific heat ratio and the gas constant. The linearized radiation-transport equation for a grey gas in local thermodynamic equilibrium with isotropic scattering taken into consideration can be written as

$$
\mu \frac{d I^{\prime}}{d x}+\left(\alpha_{a \infty, j}+\alpha_{s \infty, j}\right) I^{\prime}=\frac{\alpha_{s \infty, j}}{2} \int_{-1}^{1} I^{\prime}(x, \mu) d \mu+\frac{4 \alpha_{a \infty, j} \sigma T_{\infty, j}^{3} T^{\prime}}{\pi},
$$

where $\alpha_{a \infty, j}$ and $\alpha_{s \infty, j}$ are, respectively, the absorption and scattering coefficients referred to conditions at infinity, and $\mu \equiv \cos \theta$, with $\theta$ denoting the direction of propagation of radiation with respect to the positive $x$ axis. The radiative intensity $I^{\prime}(x, \mu)$ is related to the radiative heat flux by

$$
q^{\prime}(x)=2 \pi \int_{-1}^{1} \mu I^{\prime}(x, \mu) d \mu .
$$

Integrating (3) with respect to $\mu$ and with the aid of (1) and (4), we can rewrite (2) in the form

$$
\frac{\rho_{\infty, j} u_{\infty, j} \gamma R}{\gamma-1}\left[\frac{1-M_{\infty, j}^{2}}{1-\gamma M_{\infty, j}^{2}}\right] \frac{d T^{\prime}}{d x}-2 \pi \alpha_{a \infty, j} \int_{-1}^{1} I^{\prime}(x, \mu) d \mu+16 \alpha_{a \infty, j} \sigma T_{\infty, j}^{3} T^{\prime}=0,
$$

which together with (3) are the governing equations for the two unknowns $T^{\prime}$ and $I^{\prime}$.

At the shock $(x=0)$ the radiative intensity is continuous, that is,

$$
I^{\prime}\left(0^{-}, \mu\right)+\sigma \frac{T_{\infty, 1}^{4}}{\pi}=I^{\prime}\left(0^{+}, \mu\right)+\sigma \frac{T_{\infty, 2}^{4}}{\pi},
$$

where $0^{-}$and $0^{+}$denote conditions immediately in front of and behind the shock. At infinity $(x \rightarrow \pm \infty)$, both $T^{\prime \prime}$ and $I^{\prime}$ must vanish.

We now assume that the solution is a linear combination of terms of the form

and

$$
I^{\prime}(x, \mu)=A_{\nu}^{(j)} \exp \left[-\frac{\alpha_{\infty, j} x}{\nu}\right] \Phi_{\nu}^{(j)}(\mu) \quad(j=1,2)
$$

where $\alpha_{\infty, j} \equiv\left(\alpha_{a \infty, j}+\alpha_{s \infty, j}\right), \nu$ is the separation variable, $A_{\nu}^{(j)}$ and $C_{\nu}^{(j)}$ are expansion coefficients to be determined. The function $\Phi_{v}^{(j)}(\mu)$ remains to be found.

Substitution of (7) and (8) into (3) and (5) leads to

and

$$
A_{\nu}^{(j)}\left[1-\frac{\mu}{\nu}\right] \Phi_{\nu}^{(j)}(\mu)=\frac{1}{2} \omega_{j} A_{\nu}^{(j)} \int_{-1}^{1} \Phi_{\nu}^{(j)}(\mu) d \mu+\frac{4\left(1-\omega_{j}\right) \sigma T_{\infty, j}^{3} C_{\nu}^{(j)}}{\pi}
$$


which can be combined to give

where

$$
\left[1-\frac{\mu}{\nu}\right] \Phi_{\nu}^{(j)}(\mu)=\frac{1}{2 g_{j}(\nu)} \int_{-1}^{1} \Phi_{\nu}^{(j)}(\mu) d \mu
$$

and $\quad \omega_{j} \equiv \frac{\alpha_{s \infty, j}}{\alpha_{a \infty, j}+\alpha_{s_{\infty}, j}}, \quad \beta_{j} \equiv \frac{B o_{j}\left(1-M_{\infty, j}^{2}\right)}{16\left(1-\gamma M_{\infty, j}^{2}\right)}, \quad$ with $\quad B o_{j} \equiv \frac{\rho_{\infty, j} u_{\infty, j} R \gamma}{(\gamma-1) \sigma T_{\infty, j}^{3}}$

denoting the Boltzmann number. It is worth noting that $\beta_{1}$ is a positive quantity since $M_{\infty, 1}>1$ whereas $\beta_{2}$ can either be positive or negative, depending on $M_{\infty, 2} \lesssim 1 / \sqrt{ } \gamma$. For convenience we shall refer to the cases $M_{\infty, 2} \lesssim 1 / \sqrt{ } \gamma$ as the strong shock and the weak shock. $\omega_{j}$ is a positive quantity with magnitude less than 1 (for the case of $\omega_{j}=1$, i.e. the case of a pure scattering gas, the radiation-transport equation is decoupled from the gasdynamic equations and we have the non-radiative gasdynamic equations for the flow field; thus, in this paper, we shall consider $0 \leqslant \omega_{j}<1$ ).

\section{Method of singular eigenfunction expansions}

Except for the appearance of the function $g_{j}(\nu),(11 a)$ is similar to an integral equation in neutron-transport theory, which has been treated by Case \& Zweifel (1967). The solution of this problem can now be obtained by the method similar to the problem of neutron transport in two half spaces considered, for example. by Mendelson \& Summerfield (1964).

Since $(11 a)$ is homogeneous and with the integral representing a pure number, we can choose the normalization condition such that

so that (11a) becomes

$$
\int_{-1}^{1} \Phi_{\nu}^{(j)}(\mu) d \mu=1
$$

$$
(\nu-\mu) \Phi_{\nu}^{(j)}(\mu)=\frac{\nu}{2 g_{j}(\nu)}
$$

We now search for values of $\nu$ which give non-trivial solutions for $\Phi_{\nu}^{(j)}(\mu)$. The analysis depends upon the location of $\nu$ in the complex plane.

(i) If the value of $v$ does not lie on the real line in the interval $(-1,1),(13)$ can be solved to give

$$
\Phi_{\nu}^{(j)}(\mu)=\frac{\nu}{2 g_{j}(\nu)(\nu-\mu)}
$$

which has to be satisfied with the normalization condition (12) to give

$$
\Lambda_{j}(\nu) \equiv 1-\frac{\nu}{2 g_{j}(\nu)} \int_{-1}^{1} \frac{d \mu}{\nu-\mu}=1-\frac{\nu}{2 g_{j}(\nu)} \ln \frac{\nu+1}{\nu-1}=0 .
$$

Equation (15) is a transcendental equation for the determination of the discrete eigenvalues $\nu$. It is noted that the function $\Lambda_{j}(\nu)$ in (15) has a pole at

$$
\nu=\beta_{j} /\left(1-\omega_{j}\right)
$$


and a branch cut along the real line from -1 to 1 , and is analytic elsewhere. It follows from the Plemelj formulas (see, for example, Muskhelishvili 1953) that the boundary values of $\Lambda_{j}(\nu)$, approaching from above (+) and below (-) the cut, are

$$
\begin{aligned}
\Lambda_{j}^{ \pm}(\nu) & =1-\frac{\nu}{2 g_{j}(\nu)} P \int_{-1}^{1} \frac{d \mu}{\nu-\mu} \pm \frac{\pi i \nu}{2 g_{j}(\nu)} \\
& =1-\frac{\nu}{2 g_{j}(\nu)} \ln \frac{1+\nu}{1-\nu} \pm \frac{\pi i \nu}{2 g_{j}(\nu)} \quad(j=1,2),
\end{aligned}
$$

where $P$ denotes the principal value.

It is shown in appendix $A$ that the number of finite roots of $\Lambda_{j}(\nu)$ is either one or two, depending on the values of $\theta_{j}( \pm 1)$, where $\theta_{j}(\nu)$ is given by

$\theta_{j}(\nu)=\arg \Lambda_{j}(\nu)=\tan ^{-1}\left[\frac{\pi v / 2 g_{j}(\nu)}{1-\left(\nu / 2 g_{j}(\nu)\right) \ln [(1+\nu) /(1-v)]}\right] \quad(-1 \leqslant \nu \leqslant 1)$,

which in turn depend upon the parameters appearing in $g_{j}(\nu)$. We will not concern ourselves with the root $\nu=\infty$ since the corresponding solutions for $T^{\prime}$ and $I^{\prime}$ are constants that must vanish in order to satisfy the conditions at infinity. The result of the analysis of appendix $A$ is summarized in table 1 , where $\nu_{j i}$ denotes the discrete roots of (15), with the first subscript representing the particular medium ( $j=1$ for $x<0$, and $j=2$ for $x>0$ ) and the second subscript representing the sign of the root $(i=1$ for a negative root and $i=2$ for a positive root).

(ii) If $v$ lies on the real line between the interval $(-1,1)$, the solution of (13) is given by

$$
\Phi_{\nu}^{(j)}(\mu)=\frac{\nu}{2 g_{j}(\nu)} P \frac{1}{\nu-\mu}+\lambda_{j}(\nu) \delta(\nu-\mu),
$$

where $P$ indicates that the principal value is to be understood when integrating an expression involving $\Phi_{\nu}^{(j)}(\mu)$. The second term in (18) represents the homogeneous solution (see, for example, Lighthill 1960). The function $\Phi_{v}^{(j)}(\nu)$ given by (18) has to satisfy the normalization condition (12) which gives for $\lambda_{j}(\nu)$

$$
\lambda_{j}(\nu)=1-\frac{\nu}{2 g_{j}(\nu)} P \int_{-1}^{1} \frac{d \mu}{\nu-\mu}=1-\frac{\nu}{2 g_{j}(\nu)} \ln \frac{1+\nu}{1-\nu} .
$$

Since $\lambda_{j}(\nu)$ can always be chosen such that (19) is satisfied, any value of $v$ on the interval $(-1,1)$ is an acceptable eigenvalue. It follows from (16) and (19) that

and

$$
\begin{gathered}
\Lambda_{j}^{+}(\nu)-\Lambda_{j}^{-}(\nu)=\pi i \nu / g_{j}(\nu), \\
\Lambda_{j}^{+}(\nu)+\Lambda_{j}^{-}(\nu)=2 \lambda_{j}(\nu), \\
\Lambda_{j}^{ \pm}(\nu)=\lambda_{j}(\nu) \pm\left(\pi i \nu / 2 g_{j}(\nu)\right) \quad(j=1,2) .
\end{gathered}
$$

Thus the solution for $I^{\prime}(x, \mu)$ can be written as a superposition of the eigenfunctions given by (14) and (18) each multiplied by the exponential in $x$ as given in (7). In order to satisfy the boundary conditions at infinity $(x \rightarrow \pm \infty)$, the coefficients associated with the positive eigenvalues $(\nu>0)$ in medium 1 $(x<0)$ and the coefficients associated with negative eigenvalues $(\nu<0)$ in 


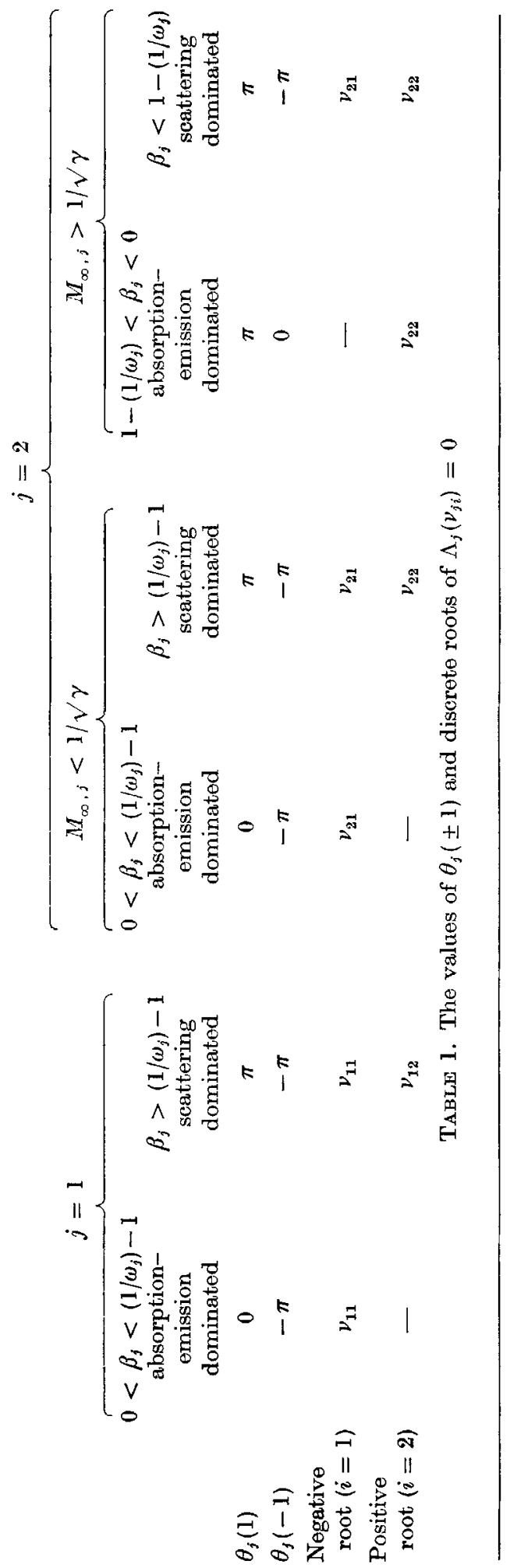


medium $2(x>0)$ must vanish. It follows from (7), (14), and (18) that, for $x>0$,

$$
I^{\prime}(x, \mu)=\left\{\begin{array}{rr}
K_{2}(1) a_{22} \Phi_{\nu, 22}^{(2)}(\mu) \exp \left[-\frac{\alpha_{\infty, 2} x}{\nu_{22}}\right]+\int_{0}^{1} \frac{\nu A_{2}(\nu)}{2 g_{2}(\nu)(\nu-\mu)} \exp \left[-\frac{\alpha_{\infty, 2} x}{\nu}\right] d \nu, \\
K_{2}(1) a_{22} \Phi_{\nu, 22}^{(2)}(\mu) \exp \left[-\frac{\alpha_{\infty, 2} x}{\nu_{22}}\right]+P \int_{0}^{1} \frac{\nu A_{2}(\nu)}{2 g_{2}(\nu)(\nu-\mu)} \exp \left[-\frac{\alpha_{\infty, 2} x}{\nu}\right] d \nu \\
+\lambda_{2}(\mu) A_{2}(\mu) \exp \left[-\frac{\alpha_{\infty, 2} x}{\mu}\right] & (\mu>0),
\end{array}\right.
$$

and, for $x<0$,

$$
I^{\prime}(x, \mu)=\left\{\begin{array}{rr}
-a_{11} \Phi_{v, 11}^{(1)}(\mu) \exp \left[-\frac{\alpha_{\infty, 1} x}{\nu_{11}}\right]-\int_{-1}^{0} \frac{\nu A_{1}(\nu)}{2 g_{1}(\nu)(\nu-\mu)} \exp \left[-\frac{\alpha_{\infty, 1} x}{\nu}\right] d \nu \\
-a_{11} \Phi_{\nu, 11}^{(1)}(\mu) \exp \left[-\frac{\alpha_{\infty} x}{\nu_{11}}\right]-P \int_{-1}^{0} \frac{\nu A_{1}(\nu)}{2 g_{1}(\nu)(\nu-\mu)} \exp \left[-\frac{\alpha_{\infty, 1} x}{\nu}\right] d \nu \\
-\lambda_{1}(\mu) A_{1}(\mu) \exp \left[-\frac{\alpha_{\infty, 1} x}{\mu}\right] & (\mu<0),
\end{array}\right.
$$

where we have introduced the notation $K_{j}( \pm 1) \equiv \pm \theta_{j}( \pm 1) / \pi$ so that the first term in $(21 a)$ and $(21 b)$ vanishes for the case when $\theta_{2}(1)=0$ (see table 1 ). Similarly, with the aid of (10), (12), and (8), the solution for $T^{\prime}$ is given by

$$
\begin{aligned}
& \left(K_{2}(1) \frac{a_{22} \pi\left(1-\omega_{2}\right) \nu_{22}}{8 \sigma T_{\infty, 2}^{3}\left[\nu_{22}\left(1-\omega_{2}\right)-\beta_{2}\right]} \exp \left[-\frac{\alpha_{\infty, 2} x}{\nu_{22}}\right]+\frac{\pi\left(1-\omega_{2}\right)}{8 \sigma T_{\infty, 2}^{3}} \int_{0}^{1} \frac{\nu A_{2}(\nu)^{*}}{\nu\left(1-\omega_{2}\right)-\beta_{2}}\right. \\
& T^{\prime}(x)=\left\{\begin{array}{r}
\times \exp \left[-\frac{\alpha_{\infty, 2} x}{\nu}\right] d \nu \quad(x>0), \quad(22 a) \\
-\frac{a_{11} \pi\left(1-\omega_{1}\right) \nu_{11}}{8 \sigma T_{\infty, 1}^{3}\left[\nu_{11}\left(1-\omega_{1}\right)-\beta_{1}\right]} \exp \left[-\frac{\alpha_{\infty, 1} x}{\nu_{11}}\right]-\frac{\pi\left(1-\omega_{1}\right)}{8 \sigma T_{\infty, 1}^{3}} \int_{-1}^{0} \frac{\nu A_{1}(\nu)}{\nu\left(1-\omega_{1}\right)-\beta_{1}}
\end{array}\right. \\
& \times \exp \left[-\frac{\alpha_{\infty, 1} x}{\nu}\right] d \nu \quad(x<0) \text {. }
\end{aligned}
$$

To obtain expressions for the coefficients $a_{j j}$ and $A_{j}(v)$ in (21) and (22), we first impose (6) on (21) to yield

$$
\Phi^{\prime}(\mu)= \begin{cases}A_{2}(\mu) \lambda_{2}(\mu)+P \int_{0}^{1} \frac{\nu A_{2}(\nu) d \nu}{2 g_{2}(\nu)(\nu-\mu)}+\int_{-1}^{0} \frac{\nu A_{1}(\nu) d \nu}{2 g_{1}(\nu)(\nu-\mu)} \quad(\mu>0), \\ A_{1}(\mu) \lambda_{1}(\mu)+\int_{0}^{1} \frac{\nu A_{2}(\nu) d \nu}{2 g_{2}(\nu)(\nu-\mu)}+P \int_{-1}^{0} \frac{\nu A_{1}(\nu) d \nu}{2 g_{1}(\nu)(\nu-\mu)} \quad(\mu<0),\end{cases}
$$

where

$$
\Phi^{\prime}(\mu)=\sigma \frac{\left(T_{\infty, 1}^{4}-T_{\infty, 2}^{4}\right)}{\pi}-K_{2}(1) a_{22} \Phi_{\nu, 22}^{(2)}(\mu)-a_{11} \Phi_{\nu, 11}^{(1)}(\mu)
$$


It is convenient to introduce the notations

$$
\begin{gathered}
A(\mu)= \begin{cases}A_{1}(\mu) & (\mu<0), \\
A_{2}(\mu) & (\mu>0),\end{cases} \\
\lambda(\mu)=\left\{\begin{array}{ll}
\lambda_{1}(\mu) & (\mu<0), \\
\lambda_{2}(\mu) & (\mu>0),
\end{array}\right\} \\
g(\mu)=\left\{\begin{array}{ll}
g_{1}(\mu) & (\mu<0), \\
g_{2}(\mu) & (\mu>0)
\end{array}\right\}
\end{gathered}
$$

Equations (23a) and $(23 b)$ can then be written as

$$
\Phi^{\prime}(\mu)=A(\mu) \lambda(\mu)+P \int_{-1}^{1} \frac{\nu A(\nu) d \nu}{2 g(\nu)(\nu-\mu)} \quad(-1 \leqslant \mu \leqslant 1) .
$$

As a first step in solving the singular integral equation (25) for $A(\nu)$, we introduce a function $n(z)$ given by

$$
n(z)=\frac{1}{2 \pi i} \int_{-1}^{1} \frac{\nu A(\nu) d \nu}{2 g(\nu)(\nu-z)}
$$

If $A(\nu) / g(\nu)$ is assumed to be sufficiently well behaved, $n(z)$ has the following properties: (i) $n(z)$ is analytic in the complex plane cut from -1 to +1 along the real axis. (ii) It goes to zero at least as fast as $1 / z$ at infinity. It follows from (26) and the Plemelj formulas that the boundary values of $n(z)$ are given by

$$
n^{+}(\mu)+n^{-}(\mu)=\frac{1}{\pi i} P \int_{-1}^{1} \frac{\nu A(\nu) d \nu}{2 g(\nu)(\nu-\mu)} \quad(-1 \leqslant \mu \leqslant 1)
$$

and

$$
n^{+}(\mu)-n^{-}(\mu)=\frac{\mu A(\mu)}{2 g(\mu)} \quad(-1 \leqslant \mu \leqslant 1) .
$$

With the aid of $(27 a),(27 b)$, and $(20 c),(25)$ can be rewritten as

where

$$
\begin{gathered}
\Lambda^{+}(\mu) n^{+}(\mu)-\Lambda^{-}(\mu) n^{-}(\mu)=\frac{\mu \Phi^{\prime}(\mu)}{2 g(\mu)}, \\
\Lambda^{ \pm}(\mu)=\Lambda_{j}^{\ddagger}(\mu) \quad\left\{\begin{array}{l}
(j=1, \quad \mu \leqslant 0), \\
(j=2, \quad \mu \geqslant 0) .
\end{array}\right\}
\end{gathered}
$$

Note that the notation for $\Lambda \pm(\mu)$, although convenient, is somewhat misleading since there exists no analytic function which has boundary values $\Lambda \pm(\mu)$.

To solve (28), it is necessary to construct a function $\chi(z)$ which is analytic in the complex plane cut from -1 to +1 along the real line with its boundary values satisfying the ratio condition

$$
\chi^{+}(\mu) / \chi^{-}(\mu)=\Lambda^{+}(\mu) / \Lambda^{-}(\mu) \quad(-1 \leqslant \mu \leqslant 1),
$$

and with the property that it is non-vanishing in the complex cut plane. It can be shown (see appendix $B$ ) that this function is given by

$$
\begin{array}{cc} 
& \chi(z)=X_{11}(z) X_{22}(z), \\
\text { where } & X_{j 2}(z) \equiv \exp \left[\frac{1}{\pi} \int_{0}^{1} \frac{\theta_{j}(t) d l}{t-z}\right] /(1-z)^{\theta_{j}(1) / \pi}, \\
\text { and } & X_{j 1}(z) \equiv \exp \left[\frac{1}{\pi} \int_{-1}^{0} \frac{\theta_{j}(t) d t}{t-z}\right] /(1+z)^{-\theta_{j}(-1) ! \pi} .
\end{array}
$$


It should be noted that $X_{j 2}(z)$ is continuous across $(-1,0)$ whereas $X_{j 1}(z)$ is continuous across $(0,1)$.

Equation (28) with the aid of (29) can therefore be written as

$$
n^{+}(\mu) \chi^{+}(\mu)-n^{-}(\mu) \chi^{-}(\mu)=\gamma(\mu) \Phi^{\prime}(\mu),
$$

where $\quad \gamma(\mu) \equiv \frac{\mu \chi^{+}(\mu)}{2 g(\mu) \Lambda^{+}(\mu)}=\frac{\mu \chi^{-}(\mu)}{2 g(\mu) \Lambda^{-}(\mu)}=\frac{1}{2 \pi i}\left[\chi^{+}(\mu)-\chi^{-}(\mu)\right]$.

The last relation in $(31 b)$ is obtained by using (20) and (29). It follows from (31 $a$ ) and the Plemelj formulas that the function $n(z)$ given by

$$
n(z)=\frac{1}{\chi(z)} \frac{1}{2 \pi i} \int_{-1}^{1} \frac{\gamma(\mu) \Phi^{\prime}(\mu) d \mu}{\mu-z}
$$

has boundary values $n^{ \pm}(\mu)$ which satisfy (31a). Substitution of (23c) into (32) leads to

$$
\begin{array}{r}
n(z)=\frac{1}{2 \pi i}\left\{\frac{\sigma\left(T_{\infty, 1}^{4}-T_{\infty, 2}^{4}\right)}{\pi}-\frac{K_{2}(1) a_{22} \nu_{22}}{2 g_{2}\left(\nu_{22}\right)\left(\nu_{22}-z\right)}-\frac{a_{11} \nu_{11}}{2 g_{1}\left(\nu_{11}\right)\left(\nu_{11}-z\right)}\right. \\
\left.+\frac{1}{\mathcal{X}(z)}\left[\frac{K_{2}(1) a_{22} \nu_{22} \chi\left(\nu_{22}\right)}{2 g_{2}\left(\nu_{22}\right)\left(\nu_{22}-z\right)}+\frac{a_{11} \nu_{11} \chi\left(\nu_{11}\right)}{2 g_{1}\left(\nu_{11}\right)\left(\nu_{11}-z\right)}\right]\right\},
\end{array}
$$

where we have used the identities (see appendix $\mathrm{C}$ )

$$
z^{l} \chi(z)=\int_{-1}^{1} \frac{\mu^{l} \gamma(\mu) d \mu}{\mu-z}, \quad\left\{\begin{array}{ll}
l=0 & \text { for } K_{2}(1)=0, \\
l=0,1 & \text { for the other cases. }
\end{array}\right\}
$$

In order that the function $n(z)$ given by (33) vanish at least as fast as $1 / z$ at infinity (i.e. property (ii)), it is required that

(i) for $K_{2}(1)=0$, we have

$$
a_{11}=\frac{2 \sigma\left(T_{\infty 1}^{4}-T_{\infty, 2}^{4}\right) g_{1}\left(\nu_{11}\right)}{\pi \nu_{11} \chi\left(\nu_{11}\right)}
$$

(ii) for all other cases, we have

and

$$
\begin{aligned}
& a_{22}=\frac{2 \sigma\left(T_{\infty, 1}^{4}-T_{\infty, 2}^{4}\right) g_{2}\left(\nu_{22}\right)}{\pi \nu_{22}\left(\nu_{11}-\nu_{22}\right) \chi\left(\nu_{22}\right)}, \\
& a_{11}=\frac{2 \sigma\left(T_{\infty, 1}^{4}-T_{\infty, 2}^{4}\right) g_{1}\left(\nu_{11}\right)}{\pi v_{11}\left(\nu_{22}-v_{11}\right) \chi\left(\nu_{11}\right)},
\end{aligned}
$$

which are obtained by expanding the function $n(z)$ in a Laurent series about infinity and letting $z \rightarrow \infty$. In arriving at (35) we have also made use of the relations

$$
\lim _{z \rightarrow \infty} \chi(z)=1 / z \text { for } \quad K_{2}(1)=0, \text { and } \lim _{z \rightarrow \infty} \chi(z)=-1 / z^{2}
$$

for other cases, which follow from (30).

With the aid of $(27 b),(33)$ and $(35)$, the coefficient $A(v)$ is given by

where

$$
\begin{gathered}
A(\nu)=L(\nu) N(\nu), \\
L(\nu) \equiv \frac{\sigma\left(T_{\infty, 1}^{4}-T_{\infty, 2}^{4}\right)}{\pi\left(\nu_{11}-\nu\right)\left(\nu_{22}-\nu\right)^{K_{2}(1)}},
\end{gathered}
$$


and

$$
N(\nu) \equiv \frac{g(\nu)}{\pi i \nu}\left[\frac{1}{\chi^{+}(\nu)}-\frac{1}{\chi^{-}(\nu)}\right]=\left\{\begin{array}{ll}
-\frac{X_{21}(\nu)}{X_{11}(\nu) Q_{2}(\nu) \Lambda_{2}^{+}(\nu) \Lambda_{2}^{-}(\nu)} & (\nu>0), \\
-\frac{X_{12}(\nu)}{X_{22}(\nu) Q_{1}(\nu) \Lambda_{1}^{+}(\nu) \Lambda_{1}^{-}(\nu)} & (\nu<0) .
\end{array}\right\}
$$

In arriving at $(36 c)$, we have made use of $(30 a),(20 a)$ and the identity (see appendix D)

$$
X_{j 1}(z) X_{j 2}(z)=Q_{j}(z) \Lambda_{j}(z)
$$

where

$$
Q_{j}(z) \equiv-\frac{z\left(1-\omega_{j}\right)-\beta_{j}}{\beta_{j}\left(1-\omega_{j}\right)\left(\nu_{j 2}-z\right)^{K_{j}(1)}\left(z-\nu_{j 1}\right)^{K_{j}(-1)}} .
$$

Equations (21) and (22) with coefficients determined from (35) and (36) are the solution to this problem. The solution is thus in terms of the function $X_{j i}(z)$ given by (30), that can be evaluated numerically.

Once the coefficients are obtained, other disturbances can be found from

$$
q^{\prime}(x)=\left\{\begin{array}{r}
K_{2}(1) 2 \pi a_{22} \nu_{22}\left[1-\frac{1}{g_{2}\left(\nu_{22}\right)}\right] \exp \left[-\frac{\alpha_{\infty, 2} x}{\nu_{22}}\right]+2 \pi\left[\int_{0}^{1} \nu A_{2}(\nu) \exp \left(-\frac{\alpha_{\infty, 2} x}{\nu}\right) d \nu\right. \\
\left.-\int_{0}^{1} \frac{\nu A_{2}(\nu)}{g_{2}(\nu)} \exp \left(-\frac{\alpha_{\infty, 2} x}{\nu}\right) d \nu\right] \quad(x>0), \quad(38 a) \\
2 \pi a_{11} \nu_{11}\left[1-\frac{1}{g_{1}\left(\nu_{11}\right)}\right] \exp \left[-\frac{\alpha_{\infty, 1} x}{\nu_{11}}\right]-2 \pi\left[\int_{-1}^{0} \nu A_{1}(\nu) \exp \left(-\frac{\alpha_{\infty, 1} x}{\nu}\right) d \nu\right. \\
\left.-\int_{-1}^{0} \frac{\nu A_{1}(\nu)}{g_{1}(\nu)} \exp \left(-\frac{\alpha_{\infty, 1} x}{\nu}\right) d \nu\right] \quad(x<0), \quad(38 b)
\end{array}\right.
$$

and

$$
\begin{aligned}
& I_{0}^{\prime}(x)=\left\{\begin{aligned}
2 \pi \int_{-1}^{1} I^{\prime}(x, \mu) d \mu=K_{2}(1) 2 \pi a_{22} \exp \left[-\frac{\alpha_{\infty, 2} x}{\nu_{22}}\right] & \\
& +2 \pi \int_{0}^{1} A_{2}(\nu) \exp \left(-\frac{\alpha_{\infty, 2} x}{\nu}\right) d \nu \quad(x>0), \\
-2 \pi a_{11} \exp \left[-\frac{\alpha_{\infty, 1} x}{\nu_{11}}\right] & -2 \pi \int_{-1}^{0} A_{1}(\nu) \exp \left[-\frac{\alpha_{\infty, 1} x}{\nu \mathrm{e}}\right] \quad(x<0) .
\end{aligned}\right. \\
& \frac{u^{\prime}}{u_{\infty, j}}=-\frac{\rho^{\prime}}{\rho_{\infty, j}}=\frac{1}{\left(1-\gamma M_{\infty, j}^{2}\right)} \frac{T^{\prime}}{T_{\infty, j}} \text {. }
\end{aligned}
$$

\section{Explicit expressions for disturbed quantities at the shock}

Simplifying expressions can be obtained for the flow and radiative quantities immediately upstream and downstream of the shock. It follows from $(21 a)$, (21c) and (6) that the radiative intensity at the shock is given by

and

$$
I^{\prime}\left(0^{+}, \mu\right)=\left\{\begin{array}{ll}
K_{2}(1) a_{22} \Phi_{\nu, 22}^{(2)}(\mu)+F(\mu) & (\mu<0), \\
-a_{11} \Phi_{\nu, 11}(\mu)-G(\mu)+(\sigma / \pi)\left(\dot{T}_{\infty, 1}^{4}-T_{\infty, 2}^{4}\right) & (\mu>0),
\end{array}\right\}
$$

$$
I^{\prime}\left(0^{-}, \mu\right)=\left\{\begin{array}{ll}
-a_{11} \Phi_{\nu, 11}^{(1)}(\mu)-G(\mu) & (\mu>0), \\
K_{2}(1) a_{22} \Phi_{\nu, 22}^{(2)}(\mu)+F(\mu)+(\sigma / \pi)\left(T_{\infty, 2}^{4}-T_{\infty, 1}^{4}\right) & (\mu<0),
\end{array}\right\}
$$




$$
\begin{array}{ll}
\text { where } & F(\mu) \equiv \int_{0}^{1} A_{2}(\nu) \Phi_{\nu}^{(2)}(\mu) d \nu=\int_{0}^{1} \frac{\nu A_{2}(\nu) d \nu}{2 g_{2}(\nu)(\nu-\mu)} \quad(\mu<0), \\
\text { and } & G(\mu) \equiv \int_{-1}^{0} A_{1}(\nu) \Phi_{\nu}^{(1)}(\mu) d \nu=\int_{-1}^{0} \frac{\nu A_{1}(\nu) d \nu}{2 g_{1}(\nu)(\nu-\mu)} \quad(\mu>0) .
\end{array}
$$

It is noted that the functions $F(\mu)$ and $G(\mu)$ have the following properties: (i) $F(z)$ is analytic in the complex plane eut from 0 to 1 and vanishes at infinity. (ii) $G(z)$ is analytic in the complex plane cut from -1 to 0 and vanishes at infinity.

We shall now obtain an explicit expression for $F(z)$. The function $G(z)$ can then be found in a similar manner. It follows from $(41 c)$ that

$$
F^{+}(\mu)-F^{-}(\mu)=\frac{\pi i \mu A_{2}(\mu)}{g_{2}(\mu)} \quad(\mu>0)
$$

which, with the aid of (36), can be written as

$$
F^{+}(\mu)-F^{-}(\mu)=-\frac{\pi i \mu}{g_{2}(\mu)} \frac{L(\mu) X_{21}(\mu)}{X_{11}(\mu) Q_{2}(\mu) \Lambda_{2}^{+}(\mu) \Lambda_{2}^{-}(\mu)} \quad(\mu>0) .
$$

We now assume that the function $F(z)$ is of the form

$$
F(z)=H(z) \frac{X_{12}(z)}{X_{22}(z)}+R(z)
$$

where $H(z)$ and $R(z)$ are analytic functions to be determined. The first term in (43) will take care of the proper discontinuity of the function $F(z)$ as given by the relation (42), whereas the second term will ensure that $F(z)$ is regular in the complex cut plane and vanishes at infinity. The function $H(z)$ can easily be obtained by comparing the discontinuity resulting from (43) with that of (42). With the aid of $(37),(20 \mathrm{c})$ and $(19)$, we obtain

$$
H(z)=\frac{C \beta_{1}\left[z\left(1-\omega_{2}\right)-\omega_{2} \beta_{2}\right]\left(\nu_{12}-z\right)^{K_{1}(1)}}{\left(1-\omega_{2}\right)\left(z-\nu_{0}\right)\left(\nu_{22}-z\right)^{K_{2}(1)}},
$$

where $\quad C \equiv \frac{\sigma\left(T_{\infty, 1}^{4}-T_{\infty, 2}^{4}\right)}{\pi\left(\beta_{2}-\beta_{1}\right)} \quad$ and $\quad \nu_{0} \equiv \frac{\beta_{1} \beta_{2}\left(\omega_{1}-\omega_{2}\right)}{\left(1-\omega_{2}\right)\left(1-\omega_{1}\right)\left(\beta_{2}-\beta_{1}\right)}$.

Thus the function $H(z)$ has a pole at $z=\nu_{0}$ when $K_{2}(1)=0$ and has poles at $z=\nu_{0}$ and $z=\nu_{22}$ when $K_{2}(1)=1$. The function $R(z)$ is obtained by requiring that $F(z)$ be regular at these values of $z$ and vanish at infinity. This yields

$$
\begin{aligned}
R(z)=-C \beta_{1}\{ & 1+\frac{X_{12}\left(\nu_{0}\right)}{X_{22}\left(\nu_{0}\right)} \frac{\left[\nu_{0}\left(1-\omega_{2}\right)-\omega_{2} \beta_{2}\right]\left(\nu_{12}-\nu_{0}\right)^{K_{1}(1)}}{\left(1-\omega_{2}\right)\left(z-\nu_{0}\right)\left(\nu_{22}-\nu_{0}\right)^{K_{2}(1)}} \\
& \left.+K_{2}(1) \frac{X_{12}\left(\nu_{22}\right)}{X_{22}\left(\nu_{22}\right)} \frac{\left[\nu_{22}\left(1-\omega_{2}\right)-\omega_{2} \beta_{2}\right]\left(\nu_{12}-\nu_{22}\right)^{K_{1}(1)}}{\left(1-\omega_{2}\right)\left(\nu_{22}-z\right)^{K_{2}(1)}\left(\nu_{22}-\nu_{0}\right)}\right\} .
\end{aligned}
$$

The function $G(z)$ can be obtained in a similar fashion by assuming the form

and we find that

$$
G(z)=M(z) \frac{X_{21}(z)}{X_{11}(z)}+S(z),
$$

$$
M(z) \equiv \frac{C \beta_{2}\left[z\left(1-\omega_{1}\right)-\omega_{1} \beta_{1}\right]\left(z-\nu_{21}\right)^{K_{2}(-1)}}{\left(1-\omega_{1}\right)\left(z-\nu_{0}\right)\left(\nu_{11}-z\right)},
$$


and $\quad S(z) \equiv C \beta_{2}\left\{1-\frac{X_{21}\left(\nu_{0}\right)}{X_{11}\left(\nu_{0}\right)} \frac{\left[\nu_{0}\left(1-\omega_{1}\right)-\omega_{1} \beta_{1}\right]\left(\nu_{0}-\nu_{21}\right)^{K_{2}(-1)}}{\left(1-\omega_{1}\right)\left(z-\nu_{0}\right)\left(\nu_{11}-\nu_{0}\right)}\right.$

$$
\left.-\frac{X_{21}\left(\nu_{11}\right)}{X_{11}\left(v_{11}\right)} \frac{\left[v_{11}\left(1-\omega_{1}\right)-\omega_{1} \beta_{1}\right]\left(\nu_{11}-\nu_{21}\right)^{K_{2}(-1)}}{\left(1-\omega_{1}\right)\left(\nu_{11}-v_{0}\right)\left(\nu_{11}-z\right)}\right\}
$$

It follows from $(22 a),(22 b)$ and (E 4) that

$$
T^{\prime}\left(0^{+}\right)=\frac{K_{2}(1) a_{22} \pi\left(1-\omega_{2}\right) \nu_{22}}{8 \sigma T_{\infty, 2}^{3}\left[\nu_{22}\left(1-\omega_{2}\right)-\beta_{2}\right]}+\frac{\pi}{8 \sigma T_{\infty, 2}^{3}} R\left(\frac{\omega_{2} \beta_{2}}{1-\omega_{2}}\right)
$$

and

$$
T^{\prime}\left(0^{-}\right)=-\frac{a_{11} \pi\left(1-\omega_{1}\right) \nu_{11}}{8 \sigma T_{\infty, 1}^{3}\left[\nu_{11}\left(1-\omega_{1}\right)-\beta_{1}\right]}-\frac{\pi}{8 \sigma T_{\infty, 1}^{3}} S\left(\frac{\omega_{1} \beta_{1}}{1-\omega_{1}}\right) .
$$

With the aid of $(38 a)$ and (E 4$)$, we have

$$
q^{\prime}(0)=K_{2}(1) 2 \pi a_{22} \nu_{22}\left[1-\frac{1}{g_{2}\left(\nu_{22}\right)}\right]-4 \pi \beta_{2} R\left(\frac{\omega_{2} \beta_{2}}{1-\omega_{2}}\right) .
$$

Making use of $(39 a),(39 b)$ and (E 5), we have

$$
I_{0}^{\prime}\left(0^{-}\right)=K_{2}(1) 2 \pi a_{22}+4 \pi g_{2}(0)\left[F(0)-\left(1-\omega_{2}\right) F\left(\frac{\omega_{2} \beta_{2}}{1-\omega_{2}}\right)\right] \quad\left(\omega_{1} \neq \omega_{2}\right),
$$

and

$$
I_{0}^{\prime}\left(0^{-}\right)=-2 \pi a_{11}-4 \pi g_{1}(0)\left[G(0)-\left(1-\omega_{1}\right) G\left(\frac{\omega_{1} \beta_{1}}{1-\omega_{1}}\right)\right] \quad\left(\omega_{1} \neq \omega_{2}\right)
$$

It is worth noting that, for the special case of $\omega_{2}=0$, the second term in (51 $\left.a\right)$ becomes indefinite (a similar situation occurs in $(51 b)$ when $\omega_{1}=0$ ). In this case, the substitution of (44) and (45) in (5l $a)$ and letting $\omega_{2} \rightarrow 0$ leads to

Similarly,

$$
\begin{aligned}
I_{0}^{\prime}\left(0^{+}\right)=K_{2}(1) & 2 \pi a_{22}-4 \pi C \beta_{1}\left\{1-\frac{X_{12}(0)}{X_{22}(0)} \frac{\beta_{2}\left(\nu_{12}\right)^{K_{1}(1)}}{\nu_{0}\left(\nu_{22}\right)^{K_{2}(1)}}\right. \\
+ & \frac{X_{12}\left(\nu_{0}\right)}{X_{22}\left(\nu_{0}\right)} \frac{\left(\beta_{2}-\nu_{0}\right)}{\nu_{0}} \frac{\left(\nu_{12}-v_{0}\right)^{K_{1}(1)}}{\left(\nu_{22}-v_{0}\right)^{K_{2}(1)}} \\
& \left.-K_{2}(1) \frac{X_{12}\left(\nu_{22}\right)}{X_{22}\left(\nu_{22}\right)} \frac{\left(\beta_{2}-\nu_{22}\right)}{\nu_{22}} \frac{\left(\nu_{12}-v_{22}\right)^{K_{1}(1)}}{\left(\nu_{22}-\nu_{0}\right)}\right\} \quad\left(\omega_{2}=0\right) .
\end{aligned}
$$

$$
\begin{aligned}
I_{0}^{\prime}\left(0^{-}\right)=-2 \pi a_{11}- & 4 \pi C \beta_{1}\left\{1-\frac{X_{21}(0)}{X_{11}(0)} \frac{\beta_{1}\left(-\nu_{21}\right)^{K_{2}(-1)}}{\nu_{0} \nu_{11}}\right. \\
& -\frac{X_{21}\left(\nu_{0}\right)}{X_{11}\left(\nu_{0}\right)} \frac{\left(\beta_{1}-\nu_{0}\right)\left(\nu_{0}-v_{21}\right)^{K_{2}(-1)}}{\nu_{0}\left(\nu_{11}-\nu_{0}\right)} \\
& \left.+\frac{X_{21}\left(\nu_{11}\right)}{X_{11}\left(\nu_{11}\right)} \frac{\left(\beta_{1}-\nu_{11}\right)}{\nu_{11}} \frac{\left(\nu_{11}-\nu_{21}\right)^{K_{2}(-1)}}{\left(\nu_{11}-\nu_{0}\right)}\right\} \quad\left(\omega_{1}=0\right) .
\end{aligned}
$$

Furthermore, for the special case when $\omega_{1}=\omega_{2}$, a limiting process must be applied to (51). The resulting expressions, however, are too complicated to be quoted here. For the numerical calculations, it is in fact easier to evaluate the expressions given by $(39 a)$ and $(39 b)$ with $x=0$ instead. 


\section{Solution based on the differential approximation}

The solution of the linearized shock wave for a non-scattering, radiating gas, based on the differential approximation and accurate to $1 / B o$, was obtained by Vincenti \& Kruger (1965). In order to compare the solution based on the differential approximation to that of the exact solution obtained in the previous sections, we shall now extend the solution of Vincenti \& Kruger to include the effect of isotropic scattering.

The differential approximation of the linearized radiation-transport equation with isotropic scattering is (Cheng 1965)

and

$$
d q^{\prime} / d x=-\alpha_{a \infty, j}\left(I_{0}^{\prime}-16 \sigma T_{\infty, j}^{3} T^{\prime}\right),
$$

These, together with (5) rewritten in terms of $I_{0}^{\prime}$, namely,

$$
{ }_{16} \sigma T_{\infty, j}^{3} \beta_{j} d T^{\prime} / d x-\alpha_{a \infty, j} I_{0}^{\prime}+16 \alpha_{a \infty, j} \sigma T_{\infty, j}^{3} T^{\prime}=0,
$$

are the governing equations for the problem. Boundary conditions at the shock are

and

$$
I_{0}^{\prime}\left(0^{-}\right)+4 \sigma T_{\infty, 1}^{4}=I_{0}^{\prime}\left(0^{+}\right)+4 \sigma T_{\infty, 2}^{4},
$$

At infinity, the disturbances are required to vanish.

The solution to the differential equations (53)-(55) with boundary conditions (56) and (57) is

$$
\begin{gathered}
\frac{T^{\prime}}{T_{\infty, j}}=B_{j j} \exp \left[-\frac{\alpha_{\infty, j} x}{\nu_{j j}}\right], \\
\frac{I_{0}^{\prime}(x)}{16 \sigma T_{\infty, j}^{4}}=\left[1-\frac{\beta_{j}}{\nu_{j j}\left(1-\omega_{j}\right)}\right] B_{j j} \exp \left[-\frac{\alpha_{\infty, j} x}{\nu_{j j}}\right], \\
\frac{q^{\prime}(x)}{4 \sigma T_{\infty, j}^{4}}=\frac{4}{3 \nu_{j j}}\left[1-\frac{\beta_{j}}{\nu_{j j}\left(1-\omega_{j}\right)}\right] B_{j j} \exp \left[-\frac{\alpha_{\infty, j} x}{\nu_{j j}}\right], \\
B_{11}=-\frac{\left[1-\left(T_{2} / T_{1}\right)^{4}\right]}{4 \nu_{22}\left(\frac{1}{\nu_{22}}-\frac{1}{\nu_{11}}\right)\left[1-\frac{\beta_{1}}{\nu_{11}\left(1-\omega_{1}\right)}\right]} \\
B_{22}=-\frac{\left[\left(T_{1} / T_{2}\right)^{4}-1\right]}{4 \nu_{11}\left(\frac{1}{\nu_{22}}-\frac{1}{\nu_{11}}\right)\left[1-\frac{\beta_{2}}{\nu_{22}\left(1-\omega_{2}\right)}\right]} .
\end{gathered}
$$

where

The constants $\nu_{j j}$ are determined from

$$
\nu_{j j}^{2}+\frac{\nu_{j j}}{3 \beta_{j}}-\frac{1}{3\left(1-\omega_{j}\right)}=0
$$

where $\nu_{11}$ is the negative root of (61) when the equation is applied to medium 1 $(x<0)$, and $\nu_{22}$ is the positive root of $(62)$ when it is applied to medium 2 . Equation (61) would be identical to the dispersion relation given by (15), after the logarithmic term in that equation is expanded in a power series for $v \gg 1$ with the first two terms in the series retained. 
The radiative intensity in the differential approximation is given by (Cheng 1966)

$$
I^{\prime}(x, \mu)=(1 / 4 \pi)\left[I_{0}^{\prime}(x)+3 \mu q^{\prime}(x)\right],
$$

where $I_{0}^{\prime}$ and $q^{\prime}$ are given, respectively, by (59) and (60). The disturbed velocity and density are given by $(40)$.

\section{Discussion of numerical results}

The exact solution for the disturbances as given by $(21),(22),(38)-(40)$, as well as the approximate solution given by (58)-(62), are computed for the following eight cases (with $\gamma=1 \cdot 4$ and $B o_{1}=10$ ):

(1) $M_{\infty, 1}=1 \cdot 1, \quad \omega_{1}=0, \quad \omega_{2}=0$;

(2) $M_{\infty, 1}=1 \cdot 1, \quad \omega_{1}=0, \quad \omega_{2}=0 \cdot 9$;

(3) $M_{\infty, 1}=1 \cdot 1, \quad \omega_{1}=0 \cdot 9, \quad \omega_{2}=0$;

(4) $M_{\infty, 1}=1 \cdot 1, \quad \omega_{1}=0.9, \quad \omega_{2}=0.9$;

(5) $M_{\infty, 1}=1 \cdot 4, \quad \omega_{1}=0, \quad \omega_{2}=0$;

(6) $M_{\infty, 1}=1 \cdot 4, \quad \omega_{1}=0, \quad \omega_{2}=0 \cdot 9$;

(7) $M_{\infty, 1}=1 \cdot 4, \quad \omega_{1}=0 \cdot 9, \quad \omega_{2}=0$;

(8) $M_{\infty, 1}=1 \cdot 4, \quad \omega_{1}=0 \cdot 9, \quad \omega_{2}=0 \cdot 9$.

This set of conditions was chosen to cover all possibilities concerning the discrete roots as shown in table 1 . In particular, cases 1-4 with

$$
M_{\infty, 2}=0.9118 \quad(0.9118>1 / \sqrt{ } \gamma)
$$

represent a weak shock, whereas cases $5-8$ with

$$
M_{\infty, 2}=0.7397 \quad(0.7397<1 / \sqrt{ } \gamma)
$$

represent a strong shock. To check the accuracy of the computations, the disturbances immediately upstream and downstream of the shock, as given by (41) and (49)-(52), are also computed.

For convenience of presentation, the disturbances are normalized to the condition at infinity downstream, namely,

$$
\begin{gathered}
T=T^{\prime} / T_{\infty, 2}, \quad u=u^{\prime} / u_{\infty, 2}, \quad I=I^{\prime} /\left(\sigma T_{\infty, 2}^{4} / \pi\right), \\
I_{0}=I_{0}^{\prime} / 4 \sigma T_{\infty, 2}^{4} \text { and } q=q^{\prime} / \sigma T_{\infty, 2}^{4} .
\end{gathered}
$$

The physical distance is referred to the mean free path of radiation, i.e. $\eta_{j}=\alpha_{\infty, j} x$, where $\eta_{j}$ is the (dimensionless) optical path length.

The values of the discrete roots, $v_{j i}$, of (15) are tabulated in table 2. For a specific shock strength and radiation level, it is shown that the discrete roots upstream of the shock, $v_{1 i}$, depend only upon the amount of scattering $\omega_{1}$ upstream and are independent of $\omega_{2}$ downstream. Similarly, the values of the $\nu_{2 i}$ depend only upon $\omega_{2}$ and are independent of $\omega_{1}$. Since the damping factor of the discrete mode is just the reciprocal of $\nu_{j i}$, we note from this table that the damping of the discrete mode decreases as the amount of scattering is increased. 
The dimensionless magnitudes of the disturbances for a normal shock wave with different shock strength and the amount of scattering are tabulated in table 3. Representative results of this table are presented in figures 1-6, where, instead of disturbed quantities, the total dimensionless quantities are plotted.

The results of cases 1 and 3 are plotted in figure 1 , which shows the effect of scattering when the gas upstream of the shock is changed from a non-scattering gas $\left(\omega_{1}=0\right)$ to a scattering-dominated gas $\left(\omega_{1}=0.9\right)$. It is shown that the average radiative intensity $\left(I_{0}\right)$ and the radiative heat flux as obtained from the exact solution and the differential approximation are continuous at the shock.

$\begin{array}{ccccc}\text { Case } & \nu_{12} & \nu_{11} & \nu_{22} & \nu_{21} \\ 1 & - & -2 \cdot 062 & 1 \cdot 195 & - \\ 2 & - & -2 \cdot 062 & 2 \cdot 240 & -1 \cdot 618 \\ 3 & 1 \cdot 228 & -2 \cdot 975 & 1 \cdot 195 & - \\ 4 & 1 \cdot 228 & -2 \cdot 975 & 2 \cdot 240 & -1 \cdot 618 \\ 5 & - & -1 \cdot 423 & - & -1 \cdot 149 \\ 6 & - & -1.423 & 1 \cdot 652 & -2 \cdot 193 \\ 7 & 1.484 & -2.445 & - & -1 \cdot 149 \\ 8 & 1.484 & -2.445 & 1 \cdot 652 & -2 \cdot 193\end{array}$

TABLE 2. Values of the discrete roots of equation (15)

The heat flux and average radiative intensity disturbances at the shock as well as the temperature and velocity disturbances immediately upstream and downstream of the shock are larger for a non-scattering gas $\left(\omega_{1}=\omega_{2}=0\right)$. Downstream of the shock where there is no scattering for both cases, the disturbances are larger for the case with $\omega_{1}=\omega_{2}=0$ than that of $\omega_{1}=0.9$ and $\omega_{2}=0$. This situation continues to hold for the region upstream near the shock. Further upstream of the shock, however, the situation is reversed, i.e. the magnitudes of the disturbances become smaller for the case of $\omega_{1}=\omega_{2}=0$, which is a direct consequence of the fact that the damping factor upstream decreases as scattering is increased, as discussed previously. A similar situation occurs if, instead of upstream, scattering of the gas downstream is increased while that of upstream remains the same, as is evident from figure 2. From figures 1 and 2 , it is noted that, regardless of the amount of scattering and the strength of the shock, the velocity disturbances are always negative ahead of the shock and positive behind the shock. The temperature disturbances, however, are always positive upstream of the shock. Downstream of the shock, the temperature disturbance is negative for $M_{\infty, 2}>1 / \sqrt{ } \gamma$ (figure 1) and positive for $M_{\infty, 2}<1 / \sqrt{ } \gamma$ (figure 2). The radiative heat flux is everywhere negative (i.e. in the direction opposite to the direction of the flow), regardless of the amount of scattering and shock strength.

By numerical integration of the exact non-linear differential equations, Pearson (1964) reported that the differential approximation yields less than $1 \%$ error in velocity for the problem of non-linear shock wave. The corresponding linearized problem as shown in figures 1 and 2 do show that the differential approximation is within $1 \%$ for velocity, temperature, and average radiative 


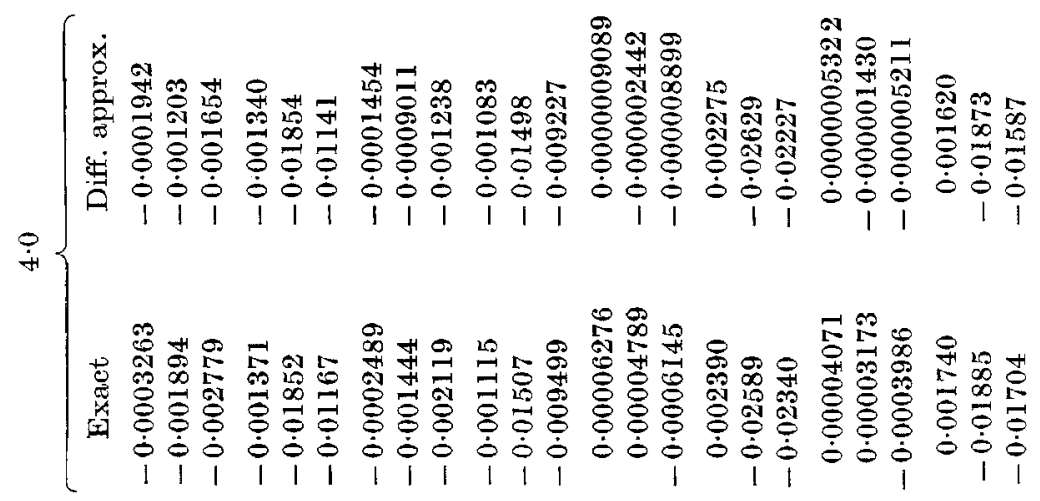

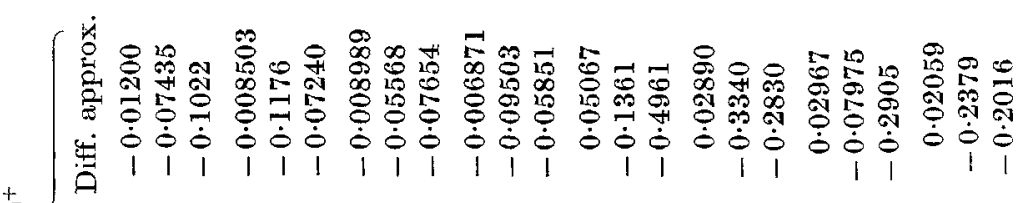
$\pm$

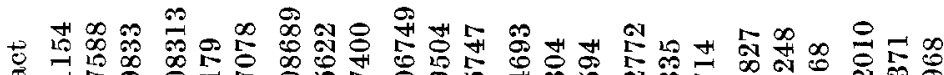
离

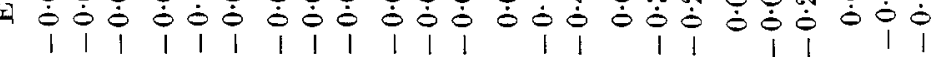
$b\{\hat{A}$

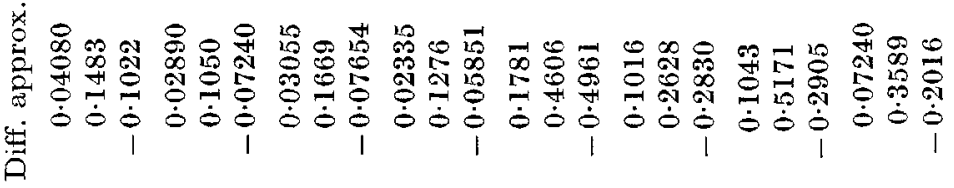

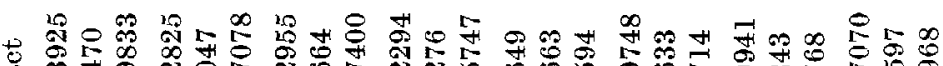
采

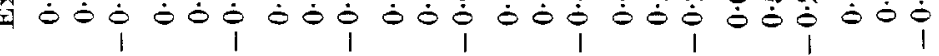

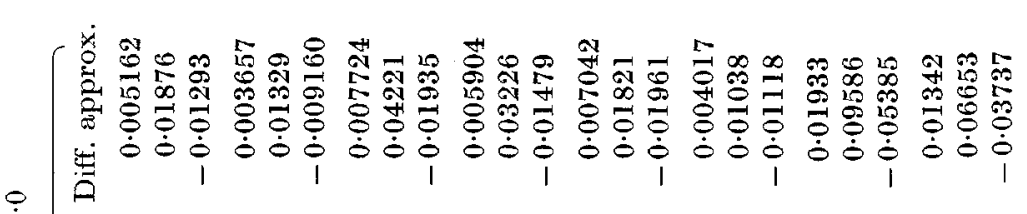

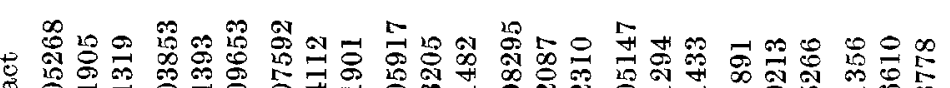

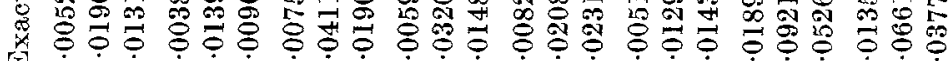

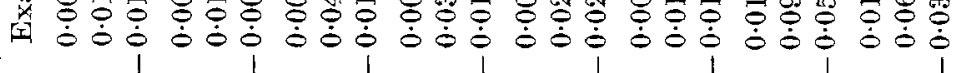

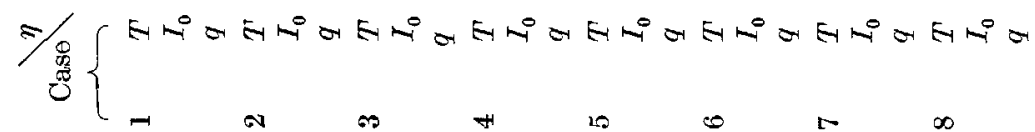

\section{莹}


intensity. The error of the radiative heat flux, however, is much larger than $1 \%$. This is due to the fact that the heat flux is a disturbed quantity while total quantities are used in the error computation for velocity, temperature, and the average radiative intensity.

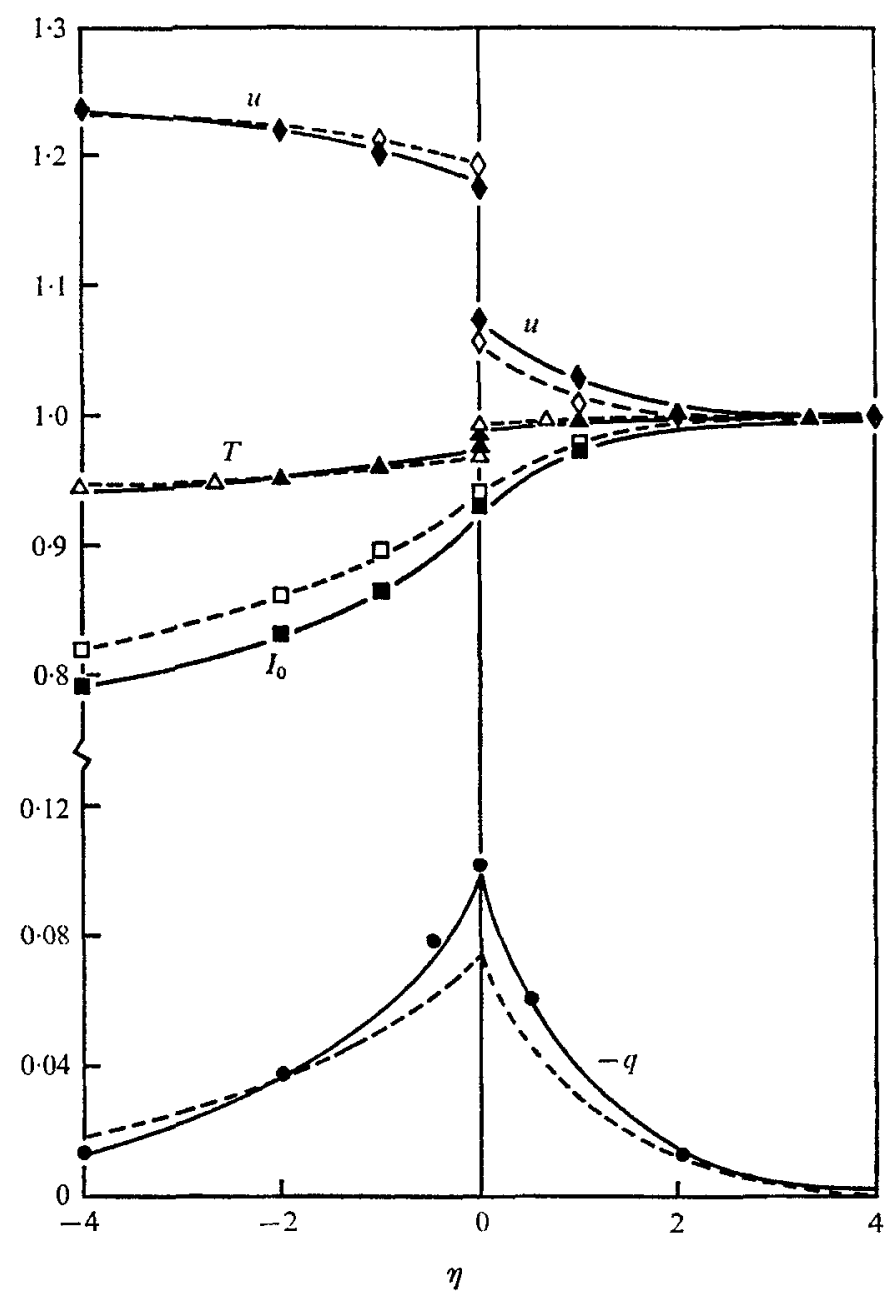

FrgURF 1. The effect of scattering in a weak normal shock wave, as obtained by the exact solution and the differential approximation $\left(M_{\infty, 1}=1 \cdot 1, M_{\infty, 2}=0.9118, \gamma=1 \cdot 4, B o_{1}=10\right)$. $\omega_{1}=\omega_{2}=0:-$ exact; $\boldsymbol{\Lambda}, \boldsymbol{\square}, \boldsymbol{O}, \boldsymbol{\sim}$, diff. approx. $\omega_{1}=0.9, \omega_{2}=0.9:---$ exact; $\triangle, \square, \bigcirc, \diamond$, diff. approx.

Intuitively, increasing the amount of isotropic scattering tends to make the radiation field more isotropic. This is indeed supported by both the exact solution and the differential approximation, as is shown in figures 3-5. Furthermore, the exact solution for the radiative intensity is shown to be discontinuous at $\theta=\frac{1}{2} \pi$ (or $\mu=0$ ), whereas the differential approximation predicts erroneously that the radiation intensity is a continuous and linear function of $\mu$, which is represented by straight lines in figure 5 . 
As is discussed in the previous section, the disturbances in general consist of a discrete mode and a continuum mode. For a strong shock with absorptionemission dominated gas downstream, however, the discrete mode disappears

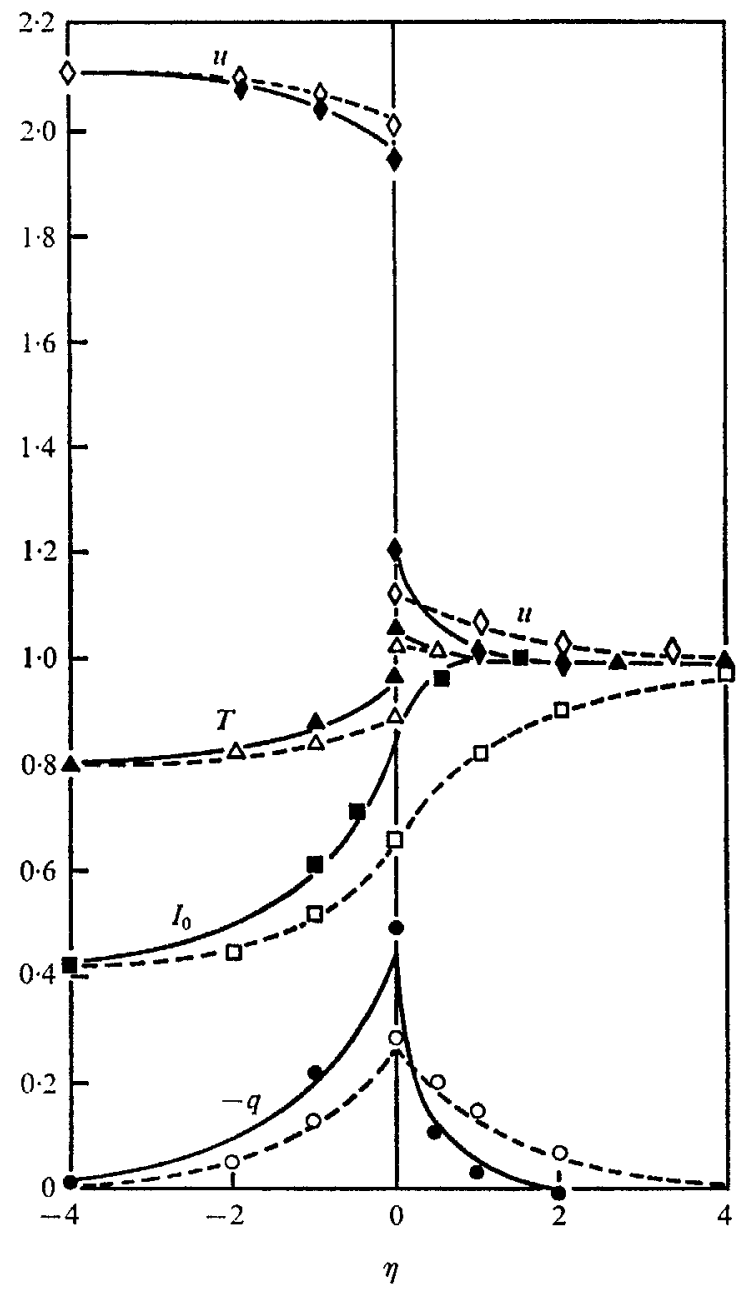

Figure 2. The effect of scattering in a strong normal shock wave, as obtained by the exact solution and the differential approximation $\left(M_{\infty 1}=1 \cdot 4, M_{\infty, 2}=0 \cdot 7397, \gamma=1 \cdot 4\right.$, $\left.B o_{1}=10\right) . \omega_{1}=\omega_{2}=0$ :

$\mathbf{\Delta}, \boldsymbol{\square}, \boldsymbol{\odot}$, diff. approx. $\omega_{1}=0, \omega_{2}=0 \cdot 9$ : -.- exact; $\triangle, \square, \bigcirc, \diamond$, diff. approx.

downstream of the shock. This situation is illustrated in figure 6 , where it is also shown that, while the magnitude of the discrete mode is greater than that of the continuum mode upstream of the shock, the damping of the discrete mode is smaller than that of the continuum mode. For this reason, only the discrete mode persists far upstream of the shock. 


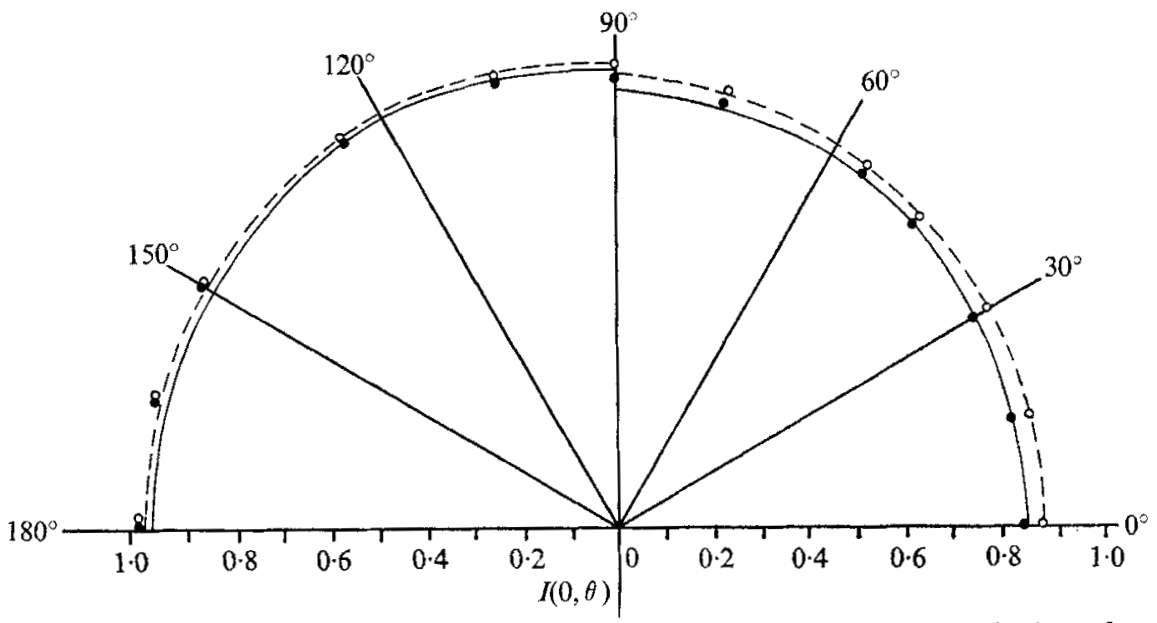

FIGURE 3. The effect of scattering on the radiative intensity (at the shock) in polar coordinates for a weak shock, as obtained by the exact solution and the differential approximation $\left(M_{\infty, 1}=1 \cdot 1, M_{\infty, 2}=0.9118, \gamma=1 \cdot 4, B o_{1}=10\right) . \omega_{1}=\omega_{2}=0:-$, exact; $\bullet$, diff. approx. $\omega_{1}=0 \cdot 9, \omega_{2}=0:---$, exact; $O$, diff. approx.

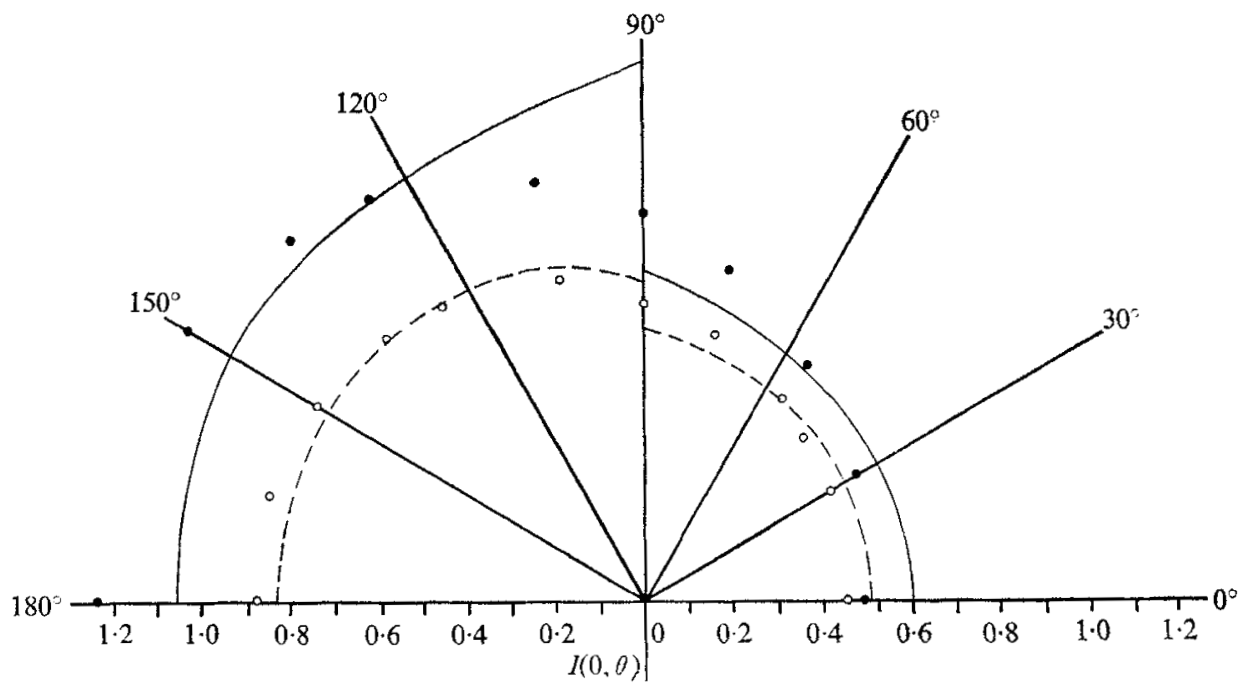

FIGURE 4. The effect of scattering on the radiative intensity (at the shock) in polar coordinates for a strong shock, as obtained by the exact solution and the differential approximation $\left(M_{\infty, 1}=1.4, M_{\infty, 2}=0.7397, \gamma=1 \cdot 4, B o_{1}=10\right) . \omega_{1}=\omega_{2}=0$ : exact; $\bullet$, diff. approx. $\omega_{1}=0, \omega_{2}=0 \cdot 9: \cdots-$, exact; $\odot$, diff. approx. 


\section{Concluding remarks}

An exact solution has been obtained for the problem of linearized flow through a normal shock wave of an emitting, absorbing, and scattering grey gas by an extension of the method of singular eigenfunction expansions. To the best of the authors' knowledge, this is the first exact solution obtained in the linearized

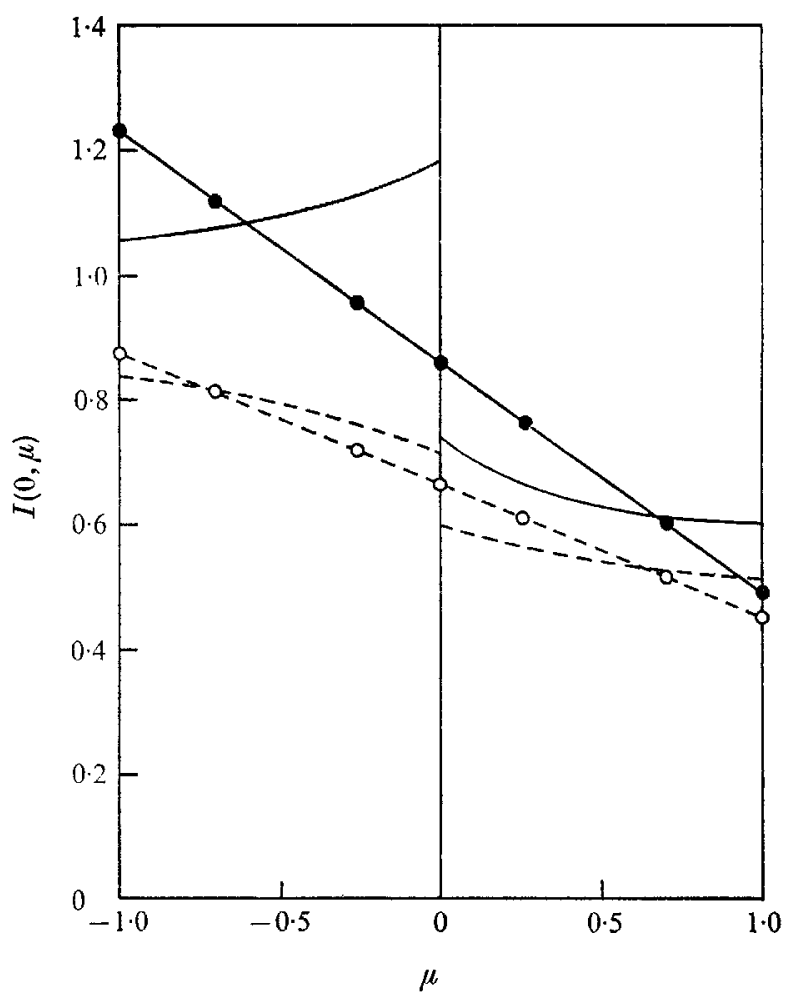

Figure 5. Radiative intensity (at the shock) as a function of $\mu$, as obtained by the exact solution and the differential approximation $\left(M_{\infty, 1}=1 \cdot 4, M_{\infty, 2}=0 \cdot 7397, \gamma=1 \cdot 4, B o_{1}=10\right)$.

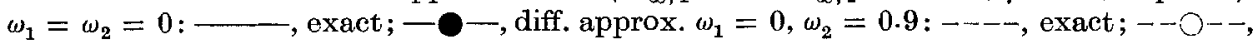
diff. approx.

analysis of a problem in radiative gasdynamics with scattering taken into consideration. The numerical results of this paper may be used as a testing case for future reference.

The effects of isotropic scattering in a normal shock wave may be summarized as follows:

The exact solution shows that, in general, the disturbances consist of a discrete mode and a continuum mode. If the gas downstream of a strong shock is absorption emission-dominated, the discrete mode disappears downstream of the shock.

When both the discrete mode and the continuum mode of disturbance exist, the amplitude of the continuum mode at the shock is smaller than that of the 
discrete mode, whereas the damping of the continuum mode is larger than that of the discrete mode. Thus only the discrete mode persists away from the shock.

Although the damping upstream $(j=1)$ depends only upon $\omega_{1}$ and is independent of $\omega_{2}$, the amplitudes of the disturbances depend both on $\omega_{1}$ and $\omega_{2}$. Similarly, although the damping downstream $(j=2)$ depends only on $\omega_{2}$ and is independent of $\omega_{1}$, the amplitudes of disturbances depend on $\omega_{1}$ and $\omega_{2}$.

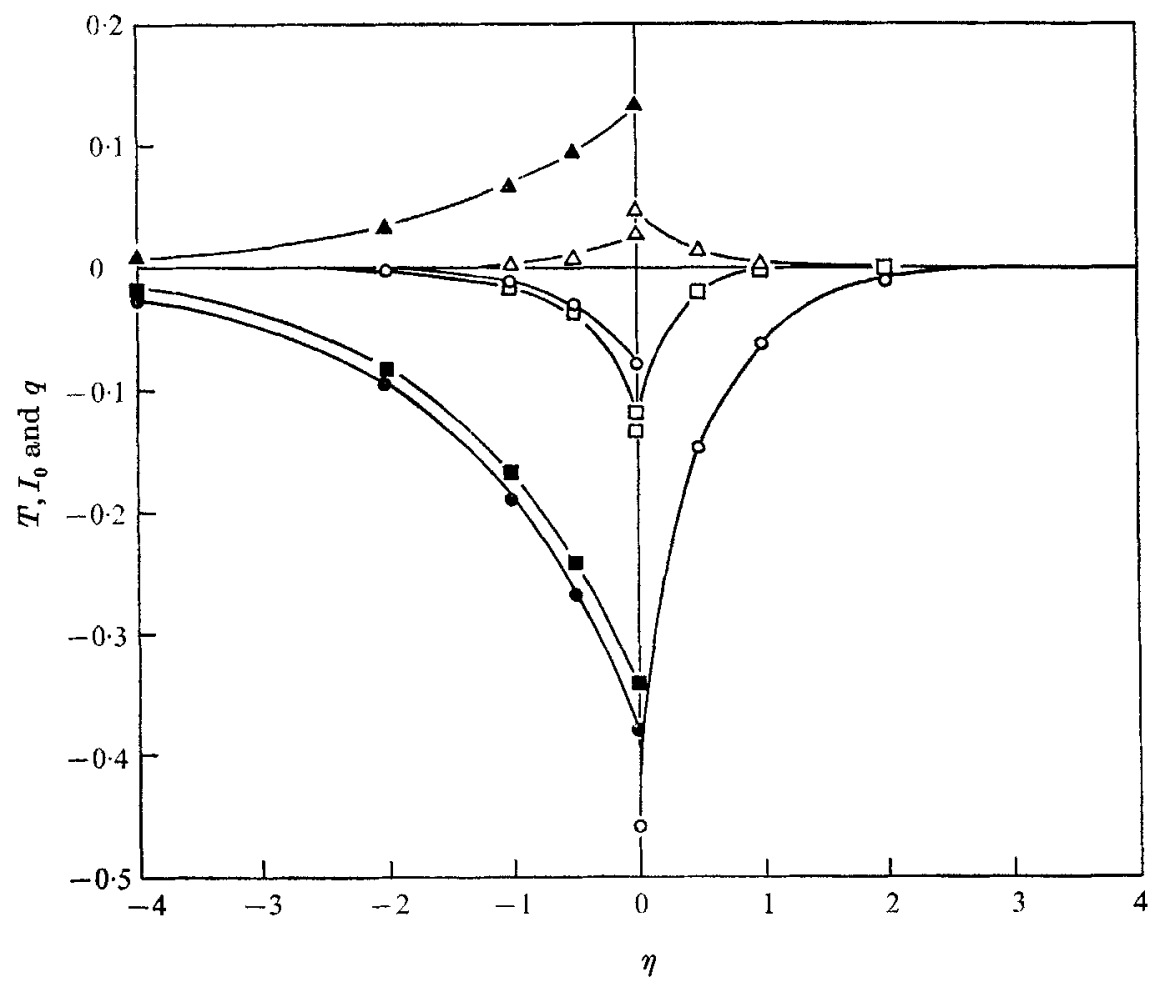

Figure 6. The contributions of the discrete and the continuum modes on the disturbances in a strong shock wave with absorption-dominated gas downstream of the shock as obtained from the exact solution $\left(M_{\infty, 1}=1 \cdot 4, M_{\infty, 2}=0 \cdot 7397, \omega_{1}=0, \omega_{2}=0, \gamma=1 \cdot 4\right.$, $B o_{1}=10$ ). Closed symbols, discrete mode; open symbols, continuum. $-\mathbf{A}-,-\triangle_{-}, T$; $-\mathrm{L}-\mathrm{-} \square-I_{0} ;-\mathrm{O}-,-\mathrm{O}-, \mathrm{q}$.

The damping of the disturbances decreases as the amount of scattering is increased in the gas.

The magnitudes of discontinuity in velocity and the temperature disturbances at the shock increase as the amount of scattering in the gas is increased.

The effect of isotropic scattering tends to make the radiation field more isotropic. Consequently, the differential approximation is more accurate as the amount of scattering in the gas is increased.

The authors would like to take this opportunity to thank M. W. Rubesin for his interest. Thanks are also due to Mrs E. Williams for her capable assistance 
in the numerical work. For the case of the first author (P.C.), this work was performed while he was in receipt of a National Academy of Sciences--National Research Council Post-doctoral Resident Associateship.

\section{Appendix A. Number of roots of equation (15)}

Consider the function $\Lambda(z)$ given by

where

$$
\Lambda(z) \equiv 1-\frac{z}{2 g(z)} \ln \frac{z+1}{z-1}
$$

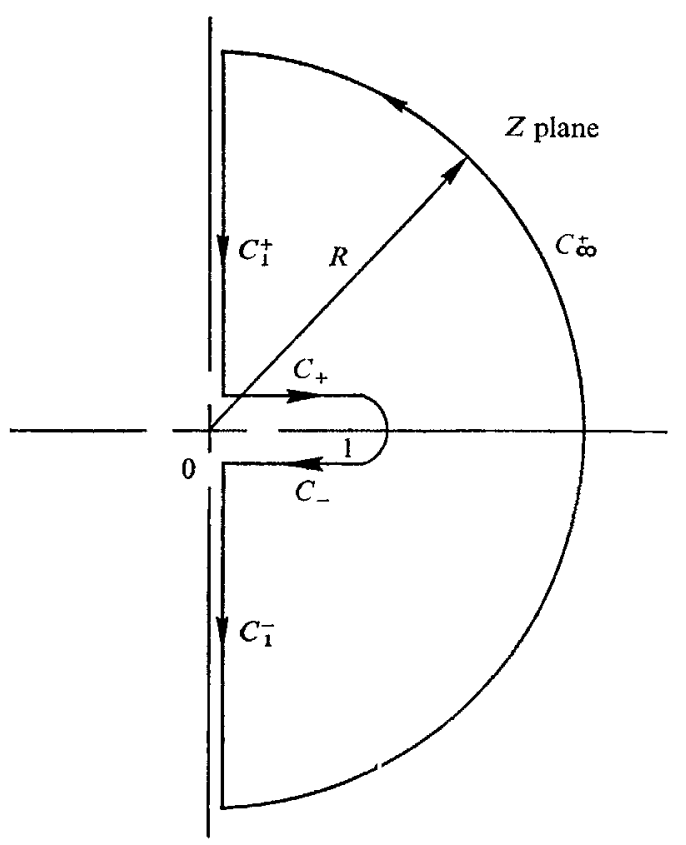

Figure 7. Sketch of the contour $C$ for equation (A4).

We wish to find the number of positive and negative zeros of $\Lambda(z)$ in the finite cut plane. To simplify the discussion we will actually consider the function $\Omega(z)$ given by

$$
\Omega(z) \equiv[\beta-(1-\omega) z] \Lambda(z) .
$$

It is noted that the function $\Lambda(z)$ has a zero at infinity whereas $\Omega(z)$ does not, and $\Lambda(z)$ has a simple pole at $z=\beta /(1-\omega)$ but $\Omega(z)$ is finite and non-zero there. Otherwise, the number and location of the finite zeros of $\Omega(z)$ are identical to those of $\Lambda(z)$.

To find, for example, the number of finite positive zeros of $\Omega(z)$, consider the closed contour in the right half cut plane as shown in figure 7. Since $\Omega(z)$ has no poles, the principle of the argument (see, for example, Copson 1955) gives the 
number of zeros of $\Omega(z)$ within the closed contour $C$ in the right half plane, $N_{z}^{R}$, as

$$
N_{z}^{R}=\left.\frac{1}{2 \pi} \Delta \arg \Omega\right|_{C}
$$

where $\left.\Delta \arg \Omega\right|_{C}$ represents the change in argument of $\Omega$ around the closed contour $C$ consisting of $C_{1}^{ \pm}, C_{ \pm}$, and $C_{\infty}$. Expanding the function $\Omega(z)$ for large $z$,

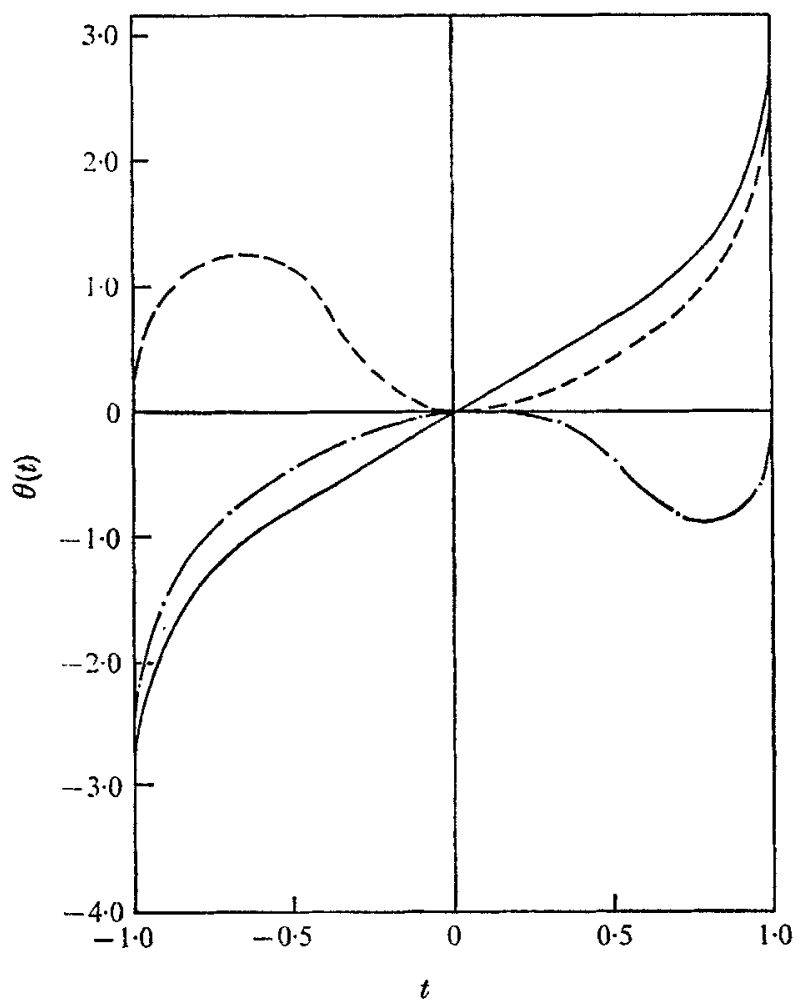

FIGURE 8. The function $\theta(t)$ versus $t$ as given by equation (A 7). - $\ldots, \beta>(1 / \omega)-I$ or $\beta<1-(1 / \omega) ; \ldots-, 0<\beta<(1 / \omega)-1 ; \ldots-\cdots, 1-(1 / \omega)<\beta<0$.

it is easy to show that the function $\Omega(z)$ is constant along $C_{\infty}(R \rightarrow \infty)$ so that the change in argument along $C_{\infty}$ is zero. Along $C_{1}^{ \pm}$(i.e. $z= \pm i k$ where $k$ is real and positive) we have

$$
\Omega( \pm i k)=\beta\left[1 \mp \omega k \tan ^{-1}(1 / k)\right] \mp i k(1-\omega)\left[1 \mp k \tan ^{-1}(1 / k)\right] .
$$

Since the real part of $\Omega( \pm i k)$ never vanishes and the imaginary part of $\Omega( \pm i k)$ vanishes at the end points of $C_{1}^{ \pm}$, and since $\arg \Omega(i k)$ is a continuous varying function of $k$, it follows that the change in argument in $\Omega(z)$ along $C_{1}^{ \pm}$is also zero.

We now consider the change in argument of $\Omega(z)$ along $C_{ \pm}$; we have

$$
\begin{aligned}
\left.(1 / 2 \pi) \Delta \arg \Omega\right|_{C_{+}+C_{-}} & =\left.(1 / 2 \pi) \Delta\left(\arg \Omega^{+}-\arg \Omega^{-}\right)\right|_{[0,1]} \\
& =(1 / \pi)[\theta(1)-\theta(0)],
\end{aligned}
$$

where

$$
\theta(t) \equiv \tan ^{-1}\left\{\frac{\pi t / 2 g(t)}{1-(t / 2 g(t)) \ln [(1+t) /(1-t)]}\right\},
$$


and we define $\theta(0) \equiv 0$. It follows from (A 4) that

$$
N_{z}^{R}=\theta(1) / \pi
$$

Similarly, one can show that the number of negative roots (i.e. roots in the finite left half plane) is given by

$$
N_{z}^{L}=-\theta(-1) / \pi
$$

Thus the number of positive and negative roots depends on the values of $\theta( \pm 1)$.

To evaluate the value of $\theta(t)$ given by (A 7) in connexion with (30b) and $(30 c)$, care must be taken to ensure that $\theta(t)$ is a continuous function and that $\theta(t)$ vanishes at $t=0$. The function $\theta(t)$ is sketched in figure 8 where it is shown that

$$
\begin{aligned}
\beta>0 ; \quad \theta(1) & =\left\{\begin{array}{ll}
\pi, & \text { if } \quad \beta>(1 / \omega)-1, \\
0, & \text { if } \quad \beta<(1 / \omega)-1,
\end{array}\right\} \\
\theta(-1) & =-\pi, \\
\beta<0 ; \quad \theta(1)=\pi, & \\
\theta(-1) & =\left\{\begin{aligned}
0, & \text { if } \beta>1-(1 / \omega), \\
-\pi, & \text { if } \beta<1-(1 / \omega) .
\end{aligned}\right\}
\end{aligned}
$$

The results of (A 8)-(A 10) as applied to upstream and downstream of the shock are given in table 1 .

\section{Appendix B. The function $\chi(z)$}

$$
\chi(z)=\frac{1}{(1-z)^{K_{2}(1)}(1+z)^{K_{1}(-1)}} \exp \left[\frac{1}{\pi} \int_{-1}^{1} \frac{\theta(t) d t}{t-z}\right],
$$

where $\theta(t)$ is given by (A 7), is analytic in the complex plane cut from -1 to +1 with its boundary values satisfying the ratio condition

$$
\chi^{+}(\mu) / \chi^{-}(\mu)=\Lambda^{+}(\mu) / \Lambda^{-}(\mu) \quad(-1<\mu<1),
$$

and is non-vanishing in the cut plane.

Proof. With the aid of the Plemelj formulas, the boundary values of $\chi(z)$ can be written as

$$
\chi^{ \pm}(\mu)=\frac{1}{(1-\mu)^{K_{2}(1)}(1+\mu)^{K_{1}(-1)}} \exp \left[\frac{P}{\pi} \int_{-1}^{1} \frac{\theta(t) d t}{t-\mu} \pm i \theta(\mu)\right] \quad(-1<\mu<1),
$$

from which we have

and consequently

$$
\ln \left[\chi^{+}(\mu) / \chi^{-}(\mu)\right]=i 2 \theta(\mu)
$$

$$
\frac{\chi^{+}(\mu)}{\chi^{-}(\mu)}=\exp [2 i \theta]=\frac{\lambda(\mu)+[\pi i \mu / 2 g(\mu)]}{\lambda(\mu)-[\pi i \mu / 2 g(\mu)]},
$$

which satisfies the ratio condition (B 2). 
To show that $\chi(z)$ is non-vanishing in the cut plane, we note that $z=-1$ and $z=1$ are the only points where the integral in (B 1) has a possibility of being infinite. Consider first the point $z=-1$ : we can rewrite $(\mathrm{B} 1)$ as

$$
\chi(z)=\frac{(-1)^{K_{1}(-1)}}{(\overline{1}-z)^{K_{2}(1)}(1-z)^{K_{1}(-1)}} \exp \left\{\frac{1}{\pi} \int_{-1}^{1} \frac{\theta(t)-\theta(-1)}{t-z} d t\right\}
$$

where the integral no longer has a logarithmic singularity at $z=-1$; consequently, $\chi(z)$ is non-vanishing at $z=-1$. Similarly, it can be shown that $\chi(z)$ is non-vanishing near $z=1$.

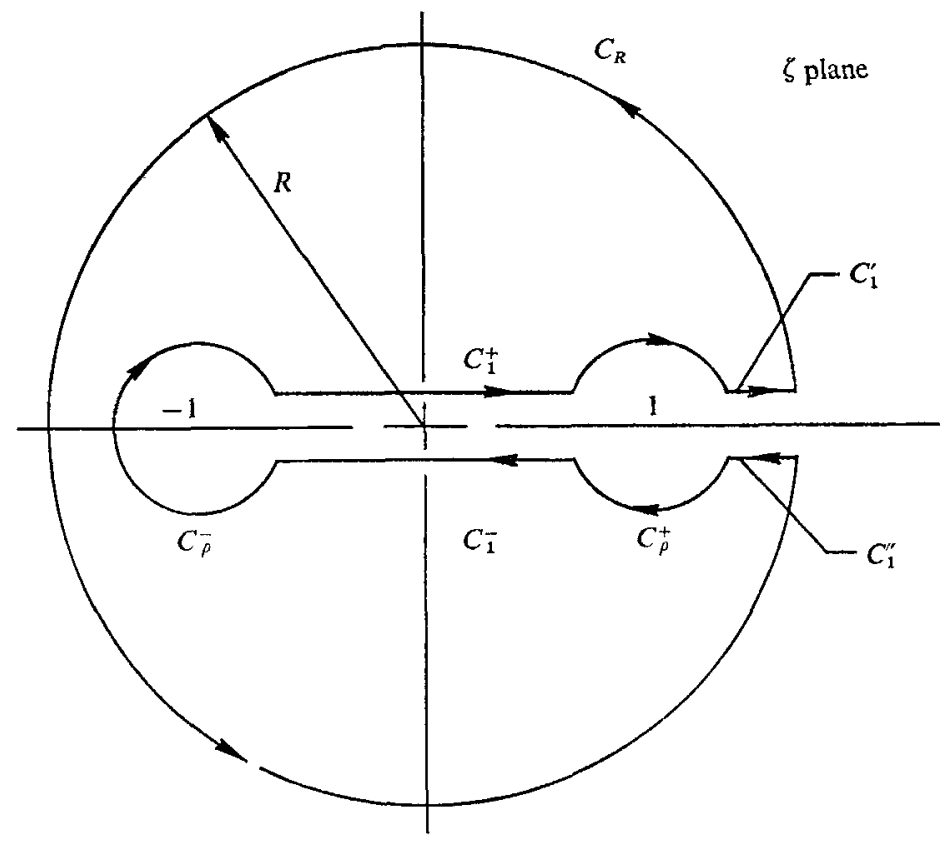

Figure 9. Sketch of the contour $C$ for equation (C2).

\section{Appendix C. Identity}

$$
z^{l} \chi(z)=\int_{-1}^{1} \frac{\mu^{l} \gamma(\mu) d \mu}{\mu-z}, \quad\left\{\begin{array}{ll}
l=0 & \text { for } K_{2}(1)=0 \\
l=0,1 & \text { for the other cases, }
\end{array}\right\}
$$

where $\gamma(\mu)$ is defined by $(31 b)$.

Proof. From Cauchy's theorem

$$
z^{l} \chi(z)=\frac{1}{2 \pi i} \int_{O} \frac{\zeta^{l} \chi(\zeta) d \zeta}{\zeta-z}
$$

where $C$ is a closed contour containing $z$ (figure 9) within which the function $\chi(z)$ is analytic. The contour $C$ consists of segments $C_{1}^{ \pm}, C_{1}^{\prime}, C_{1}^{\prime \prime}$, and curves $C_{R}$ and $C_{\rho}^{ \pm}$. Since the integrand vanishes on $C_{R}(\zeta \rightarrow \infty)$, and since the value of the integrals for $C_{\rho}^{ \pm}$are zero, it follows from (C 2) that

$$
z^{l} \chi(z)=\frac{1}{2 \pi i} \int_{-1}^{1} \frac{\mu^{l}\left[\chi^{+}(\mu)-\chi^{-}(\mu)\right] d \mu}{\mu-z},
$$


which can be written as

where we have made use of $(31 b)$.

$$
z^{l} \chi(z)=\int_{-1}^{1} \frac{\mu^{l} \gamma(\mu) d \mu}{\mu-z}
$$

\section{Appendix D. Identity}

$$
X_{j 1}(z) X_{j 2}(z)=-\frac{\left[z\left(1-\omega_{j}\right)-\beta_{j}\right] \Lambda_{j}(z)}{\beta_{j}\left(1-\omega_{j}\right)\left(\nu_{j 2}-z\right)^{K_{j}(1)}\left(z-v_{j 1}\right)^{K_{j}(-1)}} \quad(j=1,2) .
$$

Consider the function $W(z)$ defined by

$$
W(z) \equiv \frac{\Lambda_{j}(z)\left[z\left(1-\omega_{j}\right)-\beta_{j}\right]}{X_{j 1}(z) X_{j 2}(z)\left(\nu_{j 2}-z\right)^{K_{j}(1)}\left(z-\nu_{j 1}\right)^{K_{j}(-1)}} \quad(j=1,2)
$$

This function is analytic everywhere in the cut plane since both of the zeros and poles of $\Lambda_{j}(z)$ have been cancelled by appropriate factors. We now show that $W(z)$ is continuous across the cut. It follows from (D 2) that

$$
\frac{W^{+}(z)}{W^{-}(z)}=\frac{\Lambda_{j}^{+}(z) X_{\overline{j 1}}^{-}(z) X_{j 2}^{-}(z)}{\Lambda_{j}^{-}(z) X_{j 1}^{+}(z) X_{j 2}^{+}(z)}
$$

Consider for the case of $z>0$ : (D 3) reduces to

$$
\frac{W^{+}(z)}{W^{-}(z)}=1
$$

where we have made use of the fact that $X_{j 1}(z)$ is continuous for $z>0$ and (29). Similarly, it can be shown that (D 3) reduces to (D 4) for $z<0$. Thus (D 4) holds for all $z$. Furthermore, as $z \rightarrow \infty$, (D 2) gives

$$
\lim _{z \rightarrow \infty} W(z) \rightarrow-\beta_{j}\left(1-\omega_{j}\right) .
$$

Thus $W(z)$ is analytic in the cut plane, is continuous across the cut, and is equal to $-\beta_{j}\left(1-\omega_{j}\right)$ as $z \rightarrow \infty$. By Liouville's theorem, we have

or finally

$$
W(z)=-\beta_{j}\left(1-\omega_{j}\right)
$$

$$
X_{j 1}(z) X_{j 2}(z)=-\frac{\left[z\left(1-\omega_{j}\right)-\beta_{j}\right] \Lambda_{j}(z)}{\beta_{j}\left(1-\omega_{j}\right)\left(\nu_{j 2}-z\right)^{K_{j}(1)}\left(z-v_{j 1}\right)^{K_{j}(-1)}} .
$$

\section{Appendix E. Identity}

$$
\int_{0}^{1} \frac{\nu A_{2}(\nu) d \nu}{2(\nu-z)}=g_{2}(z)\left\{F(z)+\frac{\beta_{2}\left(1-\omega_{2}\right)}{\left[z\left(1-\omega_{2}\right)-\beta_{2}\right]} R\left(\frac{\omega_{2} \beta_{2}}{1-\omega_{2}}\right)\right\} .
$$

Proof. The function $F(z)$ is defined by

$$
F(z) \equiv \int_{0}^{1} \frac{\nu A_{2}(\nu) d \nu}{2 g_{2}(\nu)(\nu-z)} \quad(z<0) .
$$


Substitution of $(11 b)$ into (E 2) yields

$$
F(z)=\frac{1}{g_{2}(z)} \int_{0}^{1} \frac{\nu A_{2}(\nu) d \nu}{2(\nu-z)}-\frac{\beta_{2}\left(1-\omega_{2}\right)}{\left[z\left(1-\omega_{2}\right)-\beta_{2}\right]} \int_{0}^{1} \frac{\nu A_{2}(\nu) d \nu}{2\left[\nu-\left(\beta_{2} /\left(1-\omega_{2}\right)\right)\right]}
$$

It is noted from (42) and (E 2) that

$$
F\left(\frac{\omega_{2} \beta_{2}}{1-\omega_{2}}\right)=R\left(\frac{\omega_{2} \beta_{2}}{1-\omega_{2}}\right)=\int_{0}^{1} \frac{\nu A_{2}(\nu) d \nu}{2\left[\nu-\left(\beta_{2} /\left(1-\omega_{2}\right)\right)\right]} .
$$

Equation (E 3) with the aid of (E 4 ) reduces to (E 1), which completes the proof. By setting $z=0$ in (E 1), we obtain

$$
\int_{0}^{1} A_{2}(\nu) d \nu=2 g_{2}(0)\left[F(0)-\left(1-\omega_{2}\right) R\left(\omega_{2} \beta_{2} /\left(1-\omega_{2}\right)\right)\right],
$$

whereas, multiplying (E 1 ) by $z$ and letting $z \rightarrow \infty$, we get

$$
\int_{0}^{1} \frac{v A_{2}(v) d v}{g_{2}(\nu)}-\int_{0}^{1} \nu A_{2}(v) d \nu=2 \beta_{2} R\left(\omega_{2} \beta_{2} /\left(1-\omega_{2}\right)\right)
$$

Analogously, we can show that similar relations hold for $A_{1}(\nu), G(z)$ and $S\left(\omega_{1} \beta_{1} /\left(1-\omega_{1}\right)\right)$.

\section{REFERENCES}

Carlson, D. J. 1966 Metalized solid propellants exhaust plumes. AIAA Paper 66-652, Pt. II.

CAse, K. M. \& Zweifeu, P. F. 1967 Linearized Transport Theory. Addison-Wesley.

Cheng, P. 1965 Study of the flow of a radiating gas by a differential approximation. Ph.D. Dissertation, Stanford University.

Cheng, P. \& Leonard, A. 1970 Application of singular eigenfunction expansions to the propagation of periodic disturbances in a radiating grey gas. Submitted to Phys. Fluids.

Clarke, J. F. 1962 Structure of radiating resisted shock waves. Phys. Fluids, 5, 1347.

Copson, E. T. 1955 Theory of Functions of a Complex Variable. Oxford University Press.

Fontenot, J. E. 1965 Thermal radiation from solid rocket plumes at high altitude. A.I.A.A.J. 3, 970.

Heasler, M. A. \& Baldwin, B. S. 1963 Predictions of the structure of radiationresisted shock waves. Phys. Fluids, 6, 781 .

LighthuL, M.J. 1960 Introduction to Fourier Analysis and Generalized Functions. Cambridge University Press.

Mendelson, M. R. \& Summerfield, G. C. 1964 One-speed neutron transport in two adjacent half-spaces. $J$. Math. Phys. 5, 668 .

Mitchner, M. \& Virokur, M. 1963 Radiation smoothing of shock with and without a magnetic field. Phys. Fluids, 6, 1682.

Muskheitshvit, N. I. 1953 Singular Integral Equations. Groningen: Noordhoff.

Pearson, W. E. 1964 On the direct solution of the governing equation for radiationresisted shock wave. $N A S A T N \mathrm{D}-2128$.

Rochelse, W. C. 1967 Review of thermal radiation from liquid and solid propellant rocket exhausts. $N A S A T M X \mathbf{5 3 5 7 9}$.

Vincenti, W. G. \& Kruger, C. H. 1965 Introduction to Physical Gas Dynamics. Wiley. 